\title{
Spatially resolved cold molecular outflows in ULIRGs *
}

\author{
M. Pereira-Santaella ${ }^{1}$, L. Colina ${ }^{2}$, S. García-Burillo ${ }^{3}$, F. Combes ${ }^{4}$, B. Emonts ${ }^{5}$, S. Aalto ${ }^{6}$,

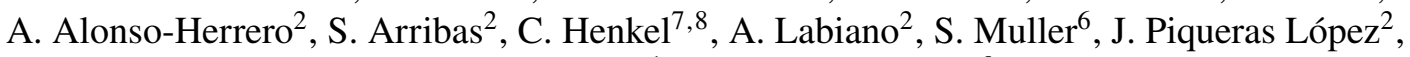 \\ D. Rigopoulou ${ }^{1}$, and P. van der Werf ${ }^{9}$
}

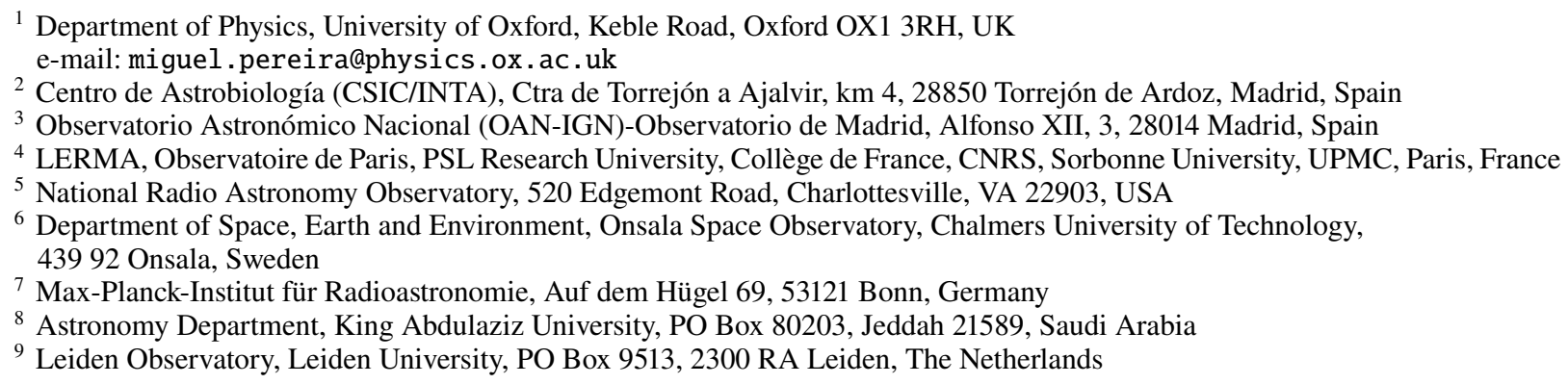

Received 23 March 2018 / Accepted 7 May 2018

\begin{abstract}
We present new $\mathrm{CO}(2-1)$ observations of three low- $z(d \sim 350 \mathrm{Mpc})$ ultra-luminous infrared galaxy (ULIRG) systems (six nuclei) observed with the Atacama large millimeter/submillimeter array (ALMA) at high spatial resolution $(\sim 500 \mathrm{pc})$. We detect massive cold molecular gas outflows in five out of six nuclei $\left(M_{\text {out }} \sim(0.3-5) \times 10^{8} M_{\odot}\right)$. These outflows are spatially resolved with deprojected effective radii between $250 \mathrm{pc}$ and $1 \mathrm{kpc}$ although high-velocity molecular gas is detected up to $R_{\max } \sim 0.5-1.8 \mathrm{kpc}$ (1-6 kpc deprojected). The mass outflow rates are $12-400 M_{\odot} \mathrm{yr}^{-1}$ and the inclination corrected average velocity of the outflowing gas is $350-550 \mathrm{~km} \mathrm{~s}^{-1}$ $\left(v_{\max }=500-900 \mathrm{~km} \mathrm{~s}^{-1}\right)$. The origin of these outflows can be explained by the strong nuclear starbursts although the contribution of an obscured active galactic nucleus cannot be completely ruled out. The position angle (PA) of the outflowing gas along the kinematic minor axis of the nuclear molecular disk suggests that the outflow axis is perpendicular to the disk for three of these outflows. Only in one case is the outflow PA clearly not along the kinematic minor axis, which might indicate a different outflow geometry. The outflow depletion times are 15-80 Myr. These are comparable to, although slightly shorter than, the star-formation (SF) depletion times $(30-80 \mathrm{Myr})$. However, we estimate that only $15-30 \%$ of the outflowing molecular gas will escape the gravitational potential of the nucleus. The majority of the outflowing gas will return to the disk after 5-10 Myr and become available to form new stars. Therefore, these outflows will not likely completely quench the nuclear starbursts. These star-forming powered molecular outflows would be consistent with being driven by radiation pressure from young stars (i.e., momentum-driven) only if the coupling between radiation and dust increases with increasing SF rates. This can be achieved if the dust optical depth is higher in objects with higher SF. This is the case in at least one of the studied objects. Alternatively, if the outflows are mainly driven by supernovae (SNe), the coupling efficiency between the interstellar medium and SNe must increase with increasing SF levels. The relatively small sizes $(<1 \mathrm{kpc})$ and dynamical times $(<3 \mathrm{Myr})$ of the cold molecular outflows suggests that molecular gas cannot survive longer in the outflow environment or that it cannot form efficiently beyond these distances or times. In addition, the ionized and hot molecular phases have been detected for several of these outflows, so this suggests that outflowing gas can experience phase changes and indicates that the outflowing gas is intrinsically multiphase, likely sharing similar kinematics, but different mass and, therefore, different energy and momentum contributions.
\end{abstract}

Key words. galaxies: active - galaxies: ISM - galaxies: kinematics and dynamics - galaxies: nuclei - galaxies: starburst

\section{Introduction}

Negative feedback from active galactic nuclei (AGN) and starbursts plays a fundamental role in the evolution of galaxies according to theoretical models and numerical simulations (e.g., Narayanan et al. 2011; Scannapieco et al. 2012; Hopkins et al. 2012; Nelson et al. 2015; Schaye et al. 2015). This feedback occurs through the injection of material, energy, and momentum into the interstellar medium (ISM) and gives rise to massive

\footnotetext{
* The reduced images and datacubes are only available at the CDS via anonymous ftp to cdsarc.u-strasbg. fr (130.79.128.5) or via http://cdsarc.u-strasbg.fr/viz-bin/qcat?J/A+A/616/A171
}

gas outflows and regulates the growth of the stellar mass and black-hole accretion.

Such energetic and massive outflows have been detected in galaxies at low and high redshift. In particular, they have been detected in ultra-luminous infrared galaxies (ULIRGs; $L_{\mathrm{IR}}>10^{12} L_{\odot}$ ) in their atomic ionized (e.g., Westmoquette et al. 2012; Arribas et al. 2014), atomic neutral (e.g., Rupke et al. 2005; Cazzoli et al. 2016), and cold molecular (e.g., Fischer et al. 2010; Feruglio et al. 2010; Sturm et al. 2011; Cicone et al. 2014) phases. Local ULIRGs are major gas-rich mergers mainly powered by star formation (SF), although AGN accounting for a significant fraction of the total infrared (IR) luminosity $(10-60 \%)$ are usually detected too (Farrah et al. 2003; 
Table 1. Sample of local ULIRGs.

\begin{tabular}{|c|c|c|c|c|c|c|c|c|c|}
\hline IRAS Name & Component & $\begin{array}{l}\text { R.A. }^{a} \\
\text { (ICRS) }\end{array}$ & $\begin{array}{c}\text { Dec. }^{a} \\
\text { (ICRS) }\end{array}$ & $\begin{array}{c}v_{\text {sys }}^{b} \\
\left(\mathrm{~km} \mathrm{~s}^{-1}\right)\end{array}$ & $z^{c}$ & $\begin{array}{c}d_{\mathrm{L}}^{d} \\
(\mathrm{Mpc})\end{array}$ & $\begin{array}{c}\text { Scale }^{d} \\
\left.(\mathrm{pc} \mathrm{arcsec})^{-1}\right)\end{array}$ & $\log {\frac{L(\mathrm{AGN})}{L_{\odot}}}^{e}$ & $\log {\frac{L_{\mathrm{IR}}}{L_{\odot}}}^{f}$ \\
\hline \multirow[t]{3}{*}{$12112+0305$} & & & & & \multirow[t]{3}{*}{0.0731} & \multirow[t]{3}{*}{330} & \multirow[t]{3}{*}{1390} & \multirow[t]{3}{*}{11.4} & \multirow[t]{3}{*}{12.19} \\
\hline & SW & $12 \mathrm{~h} 13 \mathrm{~m} 45.939 \mathrm{~s}$ & $2 \mathrm{~d} 48 \mathrm{~m} 39.10 \mathrm{~s}$ & 21167 & & & & & \\
\hline & $\mathrm{NE}$ & $12 \mathrm{~h} 13 \mathrm{~m} 46.056 \mathrm{~s}$ & $2 \mathrm{~d} 48 \mathrm{~m} 41.53 \mathrm{~s}$ & 21045 & & & & & \\
\hline \multirow[t]{3}{*}{$14348-1447$} & & & & & \multirow[t]{2}{*}{0.0825} & \multirow[t]{3}{*}{375} & \multirow[t]{2}{*}{1554} & \multirow[t]{2}{*}{11.6} & \multirow[t]{3}{*}{12.27} \\
\hline & SW & $14 \mathrm{~h} 37 \mathrm{~m} 38.280 \mathrm{~s}$ & $-15 \mathrm{~d} 00 \mathrm{~m} 24.24 \mathrm{~s}$ & 23766 & & & & & \\
\hline & $\mathrm{NE}$ & $14 \mathrm{~h} 37 \mathrm{~m} 38.396 \mathrm{~s}$ & $-15 \mathrm{~d} 00 \mathrm{~m} 21.29 \mathrm{~s}$ & 23676 & \multirow{4}{*}{0.0778} & & \multirow{4}{*}{1469} & \multirow{4}{*}{11.5} & \\
\hline \multirow{3}{*}{$22491-1808$} & & & & & & \multirow{3}{*}{353} & & & \multirow{3}{*}{12.03} \\
\hline & $\mathrm{E}$ & $22 \mathrm{~h} 51 \mathrm{~m} 49.348 \mathrm{~s}$ & $-17 \mathrm{~d} 52 \mathrm{~m} 24.12 \mathrm{~s}$ & 22412 & & & & & \\
\hline & $\mathrm{W}^{*}$ & $\cdots$ & $\cdots$ & $\cdots$ & & & & & \\
\hline
\end{tabular}

Notes. ${ }^{(a)}$ Coordinates of the $248 \mathrm{GHz}$ rest-frame continuum emission for each nucleus (see Sect. 3.1.2). The astrometric uncertainty is $\sim 25$ mas (see Sect. 3.2). ${ }^{(b)} \mathrm{CO}(2-1)$ systemic velocity using the relativistic velocity definition in the kinematic local standard of rest (LSRK; see Sect. 3.2). Typical uncertainties are $\lesssim 10 \mathrm{~km} \mathrm{~s}^{-1}$. ${ }^{(c)}$ Redshift using the average systemic velocity of the system. ${ }^{(d)}$ Luminosity distance and scale for the assumed cosmology (see Sect. 1). ${ }^{(e)}$ Luminosity of the AGN in the system estimated from mid-IR spectroscopy (Veilleux et al. 2009). ${ }^{(f)}$ IR luminosity of the system based on the SED fitting of the Spitzer and Herschel mid- and far-IR photometry (see Sect. 3.4.1). ${ }^{(*)}$ No $248 \mathrm{GHz}$ continuum is detected at the position of the near-IR W nucleus of IRAS 22491-1808.

Nardini et al. 2010). Since local ULIRGs are the hosts of the most extreme starbursts in the local Universe with star-formation rates (SFR) greater than $\sim 150 M_{\odot} \mathrm{yr}^{-1}$, based on their IR luminosities (Kennicutt \& Evans 2012), they are adequate objects to study the negative feedback from both AGN and SF. In this paper, we focus on the molecular phase of these outflows. This phase includes molecular gas with a wide range of temperatures. The hot $(T>1500 \mathrm{~K})$ and the warm $(T>200 \mathrm{~K})$ molecular phases can be observed using the near-IR ro-vibrational $\mathrm{H}_{2}$ transitions (e.g., Emonts et al. 2014, 2017; Dasyra et al. 2015) and the mid-IR rotational $\mathrm{H}_{2}$ transitions (e.g., Hill \& Zakamska 2014). However, it is thought that the energy and mass of these outflows are dominated by the cold molecular phase (e.g., Feruglio et al. 2010; Cicone et al. 2014; Saito et al. 2018), although some observations and models seem to contradict this view (e.g., Hopkins et al. 2012; Dasyra et al. 2016). The cold molecular phase has been detected using multiple $\mathrm{CO}$ transitions (e.g., Feruglio et al. 2010; Chung et al. 2011; Cicone et al. 2014; García-Burillo et al. 2015; Pereira-Santaella et al. 2016), HCN transitions (e.g., Aalto et al. 2012; Walter et al. 2017; Barcos-Muñoz et al. 2018), and far-IR OH absorption (Fischer et al. 2010; Sturm et al. 2011; Spoon et al. 2013; González-Alfonso et al. 2017). All these observations have revealed that cold molecular outflows are common in ULIRGs and that they can be massive enough to play a relevant role in the regulation of the SF in their host galaxies.

Knowing the distribution of the outflowing gas is important to derive accurate outflow properties, like the outflow mass, energy, and momentum rates, which are key to determine the impact of these outflows onto their host galaxies. However, spatially resolved observations of outflows in ULIRGs are still limited to a few sources (e.g., García-Burillo et al. 2015; Veilleux et al. 2017; Barcos-Muñoz et al. 2018; Saito et al. 2018). Here, we present new high angular resolution ( 0.'3-0.'4) Atacama large millimeter/submillimeter array (ALMA) observations of the $\mathrm{CO}(2-1)$ transition in three low- $z$ ULIRGs where the cold molecular outflow phase is spatially resolved on scales of $\sim 500 \mathrm{pc}$. This provides a direct measurement of the outflow size and therefore allows us to derive more accurately the outflow properties.

This paper is organized as follows: the sample and the ALMA observations are described in Sect. 2. In Sect. 3, we analyze the $248 \mathrm{GHz}$ continuum and $\mathrm{CO}(2-1)$ emissions and measure the outflow properties in these systems. The energy source of the outflows, as well as their impact, launching mechanism, and multi-phase structure are discussed in Sect. 4. Finally, in Sect. 5, we summarize the main results of the paper. Throughout this article we assume the following cosmology: $H_{0}=70 \mathrm{~km} \mathrm{~s}^{-1} \mathrm{Mpc}^{-1}, \Omega_{m}=0.3$, and $\Omega_{\Lambda}=0.7$.

\section{Observations and data reduction}

\subsection{Sample of ULIRGs}

In this paper, we study three low- $z(d \sim 350 \mathrm{Mpc})$ ULIRGs (six individual nuclei) with $\log L_{\mathrm{IR}} / L_{\odot}=12.0-12.3$ (see Table 1) based on their mid- and far-IR spectral energy distribution modeling (Sect. 3.4.1). These three ULIRG systems seem to be in a similar dynamical state. They were classified as type III by Veilleux et al. (2002), which corresponds to a pre-merger stage characterized by two identifiable nuclei with well defined tidal tails and bridges. They also belong to the subclass of "close binary" (i.e., "b") as the projected separation of their nuclei is smaller than $10 \mathrm{kpc}$.

The nuclei of these galaxies are classified as low ionization nuclear emission-line regions (LINER; IRAS 12112+0305 and IRAS 14348-1447; e.g., Colina et al. 2000; Evans et al. 2002) or H II (IRAS 22491-1808; e.g., Veilleux et al. 1999) and in all systems a weak AGN contribution (10-15\%) is detected in their mid-IR Spitzer spectra (Veilleux et al. 2009). For IRAS 14348-1447, high-angular-resolution mid-IR imaging suggests that the AGN is located in the southwest nucleus (Alonso-Herrero et al. 2016). For IRAS 12112+0305 and IRAS 22491-1808, we assume that the AGN is at the brightest nucleus in the radio/sub-mm continuum, that is, IRAS 12112+0305 NE and IRAS 22491-1808 E (see below). In addition, vibrationally excited $\mathrm{HCN} J=4-3$ emission is detected in IRAS 12112+0305 NE and IRAS 22491-1808 E, which can be a signature of hot dust heated by an AGN (Imanishi et al. 2016, 2018). In addition, these three ULIRGs belong to a representative sample of local ULIRGs studied by García-Marín et al. (2009a,b), Arribas et al. (2014), and Piqueras López et al. (2012) using optical and near-IR integral field spectroscopy. 
Table 2. ALMA observation log.

\begin{tabular}{|c|c|c|c|c|c|c|c|c|}
\hline \multirow[t]{2}{*}{ Object } & \multirow[t]{2}{*}{ Date } & \multirow{2}{*}{$\begin{array}{c}\text { Observed } \\
\text { frequency } \\
(\mathrm{GHz})\end{array}$} & \multirow{2}{*}{$\begin{array}{c}\text { On-source } \\
\text { time } \\
(\mathrm{min}) \\
\end{array}$} & \multirow{2}{*}{$\begin{array}{c}\text { Maximum } \\
\text { recoverable scale }\end{array}$} & \multirow{2}{*}{$\begin{array}{l}\text { Synthesized } \\
\text { beam }^{b}\end{array}$} & \multicolumn{2}{|c|}{ Amplitude calibrator } & \multirow{2}{*}{$\begin{array}{l}\text { Sensitivity }^{c} \\
\left(\mu \mathrm{Jy} \mathrm{beam}^{-1}\right)\end{array}$} \\
\hline & & & & & & Name & $\begin{array}{c}\text { Flux } \\
(\mathrm{Jy})\end{array}$ & \\
\hline $12112+0305$ & $\begin{array}{l}2017-05-08 \\
2017-05-09\end{array}$ & 214.81 & 37 & $3 . .9$ & $0.36 \times 0 . .27,-82^{\circ}$ & $\mathrm{J} 1229+0203$ & $7.01 \pm 0.27$ & $310 / 33$ \\
\hline $14348-1447$ & $\begin{array}{l}2017-05-09 \\
2017-05-22\end{array}$ & 212.87 & 39 & 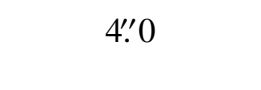 & $0.32 \times 0 . .26,-78^{\circ}$ & $\mathrm{J} 1517-2422$ & $\begin{array}{l}1.79 \pm 0.11 \\
2.15 \pm 0.15\end{array}$ & $340 / 32$ \\
\hline $22491-1808$ & $\begin{array}{l}2016-06-21 \\
2016-07-21\end{array}$ & 213.92 & 29 & $4 .{ }^{\prime \prime} 0$ & $0.48 \times 0 . .34,-84^{\circ}$ & Pallas & Butler $^{d}$ & $450 / 46$ \\
\hline
\end{tabular}

Notes. ${ }^{(a)}$ Central observed frequency of the $\mathrm{CO}(2-1)$ spectral window. ${ }^{(b)} \mathrm{FWHM}$ and position angle of the synthesized beam using Briggs weighting with a robustness parameter of $0.5 .{ }^{(c)} 1 \sigma$ line/continuum sensitivities after combining the two scheduling blocks for each object. For the line sensitivity, we use the $7.8 \mathrm{MHz}\left(\sim 10 \mathrm{~km} \mathrm{~s}^{-1}\right)$ channels of the final data cube. ${ }^{(d)}$ Flux estimated using the Butler-JPL-Horizons 2012 models and ephemeris information (see ALMA Memo \#594).

\subsection{ALMA data}

We obtained Band 6 ALMA CO(2-1) $230.5 \mathrm{GHz}$ and continuum observations for these three local ULIRGs (see Table 1) as part of the ALMA projects 2015.1.00263.S and 2016.1.00170.S (PI: Pereira-Santaella). The observations were carried out between June 2016 and May 2017. The total on-source integration times per source were $\sim 30-40$ min split into two scheduling blocks. The baseline lengths range between 15 and $1100 \mathrm{~m}$ providing a synthesized beam full width at half maximum (FWHM) of 0.'3-0.'4 (400-500 pc at the distance of these ULIRGs). Details on the observations for each source are listed in Table 2.

Two spectral windows of $1.875 \mathrm{GHz}$ bandwidth $\left(0.976 \mathrm{MHz} \equiv \sim 1.3 \mathrm{~km} \mathrm{~s}^{-1}\right.$ channels) were centered at the sky frequency of the ${ }^{12} \mathrm{CO}(2-1)$ and $\mathrm{CS}(5-4)$ transitions (see Table 2). In addition, a continuum spectral window was set at $\sim 248 \mathrm{GHz}(\sim 1.2 \mathrm{~mm})$. In this paper, we analyze the $\mathrm{CO}(2-1)$ and continuum spectral windows; the $\mathrm{CS}(5-4)$ data will be presented in a future paper.

The data were calibrated using the ALMA reduction software Common Astronomy Software Applications (CASA v4.7; McMullin et al. 2007). The amplitude calibrators for each scheduling block are listed in Table 2. For the $\mathrm{CO}(2-1)$ spectral window, a constant continuum level was estimated using the line free channels and then subtracted in the $u v$-plane. For the image cleaning, we used the Briggs weighting with a robustness parameter of 0.5 (Briggs 1995). The synthesized beam $(\sim 0 ! 3-0 ., 4)$ and maximum recoverable scale $\left(\sim 4^{\prime \prime}\right)$ are presented in Table 2 for each observation. To our knowledge, there are no single-dish $\mathrm{CO}(2-1)$ fluxes published for these ULIRGs so it is not straightforward to estimate if we filter part of the extended emission. However, the bulk of the $\mathrm{CO}(2-1)$ emission of these systems is relatively compact (see Sect. 3.1.1 and Figs. B.1-B.3 in Appendix B), so we expect to recover most of the $\mathrm{CO}(2-1)$ emission with these array configurations. The final datacubes have $300 \times 300$ spatial pixels of 0 .' 08 and 220 spectral channels of $7.81 \mathrm{MHz}\left(\sim 10 \mathrm{~km} \mathrm{~s}^{-1}\right)$. For the $\mathrm{CO}(2-1)$ cubes, the $1 \sigma$ sensitivity is $\sim 310-450 \mu \mathrm{Jy}$ beam $^{-1}$ per channel and $\sim 30-45 \mu \mathrm{Jy}$ beam $^{-1}$ for the continuum images. A primary beam correction $\left(F W H M \sim 20^{\prime \prime}\right)$ was applied to the data.

\subsection{Near-IR HST imaging}

We downloaded the near-IR Hubble space telescope/Near Infrared Camera and Multi-Object Spectrometer (HST/
NICMOS) F160W $\left(\lambda_{\mathrm{c}}=1.60 \mu \mathrm{m}, F W H M=0.34 \mu \mathrm{m}\right)$ and $\mathrm{F} 222 \mathrm{M}\left(\lambda_{\mathrm{c}}=2.21 \mu \mathrm{m}, F W H M=0.15 \mu \mathrm{m}\right)$ reduced images from the Mikulski Archive for Space Telescopes (MAST). The angular resolutions of these images are 0.14 and 0.20 for the F160W and F222M filters, respectively, which is slightly better than the resolution of the ALMA data. The ALMA and HST images were aligned using the positions of the nuclei in the $248 \mathrm{GHz}$ and F222M images. The F222M filter was used because it is less affected by dust obscuration than F160W. If the $2.2 \mu \mathrm{m}$ near-IR and $248 \mathrm{GHz}$ continua have similar spatial distributions in these ULIRGs, the uncertainty of the image alignment is about $0.08(\sim 120 \mathrm{pc})$ limited by the centroid accuracy in the HST data.

\section{Data analysis}

\subsection{Morphology}

In Figs. 1-3, we present the $\mathrm{CO}(2-1)$ and $248 \mathrm{GHz}$ continuum emission maps of the three ULIRGs.

\subsubsection{Molecular gas}

The molecular gas traced by the $\mathrm{CO}(2-1)$ transition, which is dominated by the emission from the central $\sim 1-2 \mathrm{kpc}$, has an irregular morphology with multiple large-scale tidal tails (up to $\sim 10 \mathrm{kpc}$ ) and isolated clumps. These characteristics are very likely connected to the ongoing galaxy interactions taking place in these systems. Similar tidal tails are observed in the stellar component in the near-IR HST/NICMOS NIC2 images (righthand panel of Figs. 1-3). However, there are noticeable offsets, $\sim 1-2 \mathrm{kpc}$, between the position of these stellar and molecular tidal tails.

To measure the total $\mathrm{CO}(2-1)$ emission of each system, we first defined the extent of this emission by selecting all the contiguous pixels where the $\mathrm{CO}(2-1)$ line peak is above $6 \sigma$ (see second panel of Figs. 1-3). Then, we integrated the line flux in this area. The resulting flux densities are presented in Table 3. The flux strongly peaks at the nuclei of these objects, so we calculate an effective radius based on the area, $A$, which encloses half of the total $\mathrm{CO}(2-1)$ emission as $R_{\text {eff }}=\sqrt{A / \pi}$. This $R_{\text {eff }}$ provides a better estimate of the actual size of the $\mathrm{CO}(2-1)$ emission. For these galaxies, the effective radius varies between $400 \mathrm{pc}$ and $1 \mathrm{kpc}$ (see Table 3 ).

Both in IRAS 12112 and IRAS 22491, the CO(2-1) emission is completely dominated by one of the galaxies that produces 80 

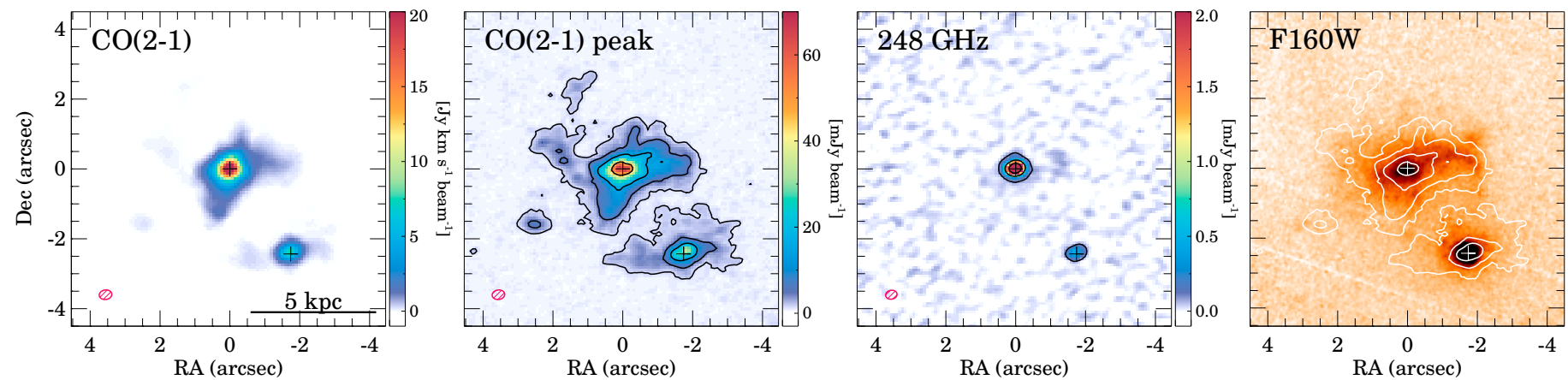

Fig. 1. ALMA and HST maps for IRAS $12112+0305$. The first and second panels are the $\mathrm{CO}(2-1)$ integrated flux and peak intensity for $\sim 10 \mathrm{~km} \mathrm{~s}^{-1}$ channels, respectively. The contour levels in the second panel correspond to $(6,18,54,162,484) \times \sigma$, where $\sigma$ is the line sensitivity (Table 2 ). The third panel is the ALMA $248 \mathrm{GHz}$ continuum. The contours in this panel are $(3,27,81) \times \sigma$ where $\sigma$ is the continuum sensitivity (Table 2). The fourth panel shows the near-IR HST/NICMOS F160W map with the $\mathrm{CO}(2-1)$ peak contours. The position of the two nuclei is marked with a cross in all the panels. The red hatched ellipse represents the FWHM and PA of the ALMA beam (Table 2).
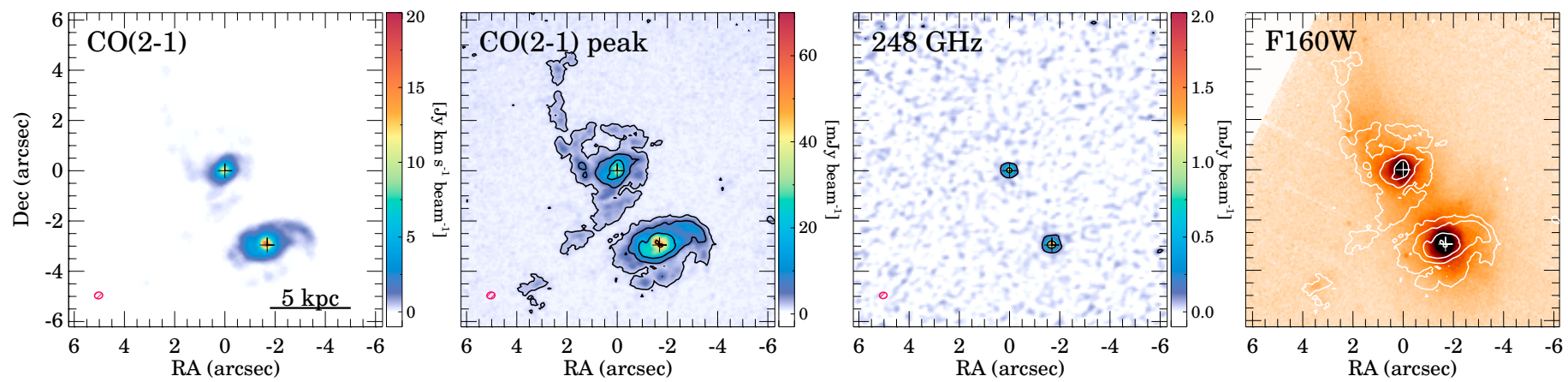

Fig. 2. Same as Fig. 1 but for IRAS 14348-1447.

Table 3. Integrated $\mathrm{CO}(2-1)$ emission.

\begin{tabular}{lcccc}
\hline \hline Object & $\begin{array}{c}S_{\mathrm{CO}}{ }^{a} \\
\left(\mathrm{Jy} \mathrm{km} \mathrm{s}^{-1}\right)\end{array}$ & $\begin{array}{c}\text { Total size }^{b} \\
\left(\mathrm{kpc}^{2}\right)\end{array}$ & $\begin{array}{c}R_{\text {eff }}{ }^{c} \\
(\operatorname{arcsec})\end{array}$ & $(\mathrm{pc})$ \\
\hline I12112 SW & 24.5 & $9.3 \pm 0.3$ & 0.31 & $430 \pm 30$ \\
I12112 NE & 117.2 & $16.5 \pm 0.4$ & 0.41 & $570 \pm 30$ \\
I14348 SW & 105.9 & $18.9 \pm 0.5$ & 0.50 & $780 \pm 40$ \\
I14348 NE & 53.3 & $19.6 \pm 0.5$ & 0.38 & $590 \pm 40$ \\
I22491 E & 59.4 & $8.3 \pm 0.3$ & 0.30 & $450 \pm 30$ \\
I22491 W & 4.1 & $7.0 \pm 0.3$ & 0.81 & $1100 \pm 40$ \\
\hline
\end{tabular}

Notes. ${ }^{(a)}$ Total $\mathrm{CO}(2-1)$ flux. The absolute flux uncertainty is $\sim 10 \%$.

${ }^{(b)}$ Size of the area where the $\mathrm{CO}(2-1)$ emission is detected at $>6 \sigma$. This is calculated as the number of pixels with emission $>6 \sigma$ multiplied by the projected pixel area on the sky. The uncertainties are the square root of the number of pixels times the projected pixel area. ${ }^{(c)}$ Effective radius of the region that encloses $50 \%$ of the total $\mathrm{CO}(2-1)$ emission defined as $R_{\mathrm{eff}}=\sqrt{A / \pi}$, where $A$ is the area of this region.

and $90 \%$, respectively, of the total flux of the merging system. In IRAS 14348 , the $\mathrm{CO}(2-1)$ emission is also dominated by one of the nuclei (SW), but this one is only two times brighter than the NE nucleus.

\subsection{2. $248 \mathrm{GHz}$ continuum}

Except for the western nucleus of IRAS 22491, which is not seen at $248 \mathrm{GHz}$, the remaining nuclei are clearly detected in both the $\mathrm{CO}(2-1)$ and continuum images. In all the cases, the $248 \mathrm{GHz}$ continuum emission is produced by a relatively compact source.
To accurately measure the continuum properties, we used the UVMULTIFIT library (Martí-Vidal et al. 2014) within CASA. This library can simultaneously fit various models to the visibility data. First, we tried a two-dimensional (2D) circular Gaussian model, which provided a good fit for all the sources except two (I12112 NE and I22491 E). For these two sources, we added a delta function with the same center as a second component to account for the unresolved continuum emission. This second unresolved component represents $70-80 \%$ of the total continuum emission in these objects. The results of the fits are presented in Table 4 and the center coordinates listed in Table 1 (see also Appendix A).

The resolved continuum emission (2D circular Gaussian component of the model) has a FWHM between 260 and $1000 \mathrm{pc}$, which is more compact than the $\mathrm{CO}(2-1)$ emission. For comparison, the $\mathrm{CO}(2-1)$ effective radius is three to six times larger than the $F W H M / 2$ of this 2D Gaussian component. Only in I22491 E, both have similar sizes, although, in this galaxy, the continuum emission is dominated by the unresolved component. Therefore, in all these ULIRGs, the $248 \mathrm{GHz}$ continuum emission is considerably more compact than the molecular $\mathrm{CO}(2-1)$ emission. This is similar to what is observed in other local luminous IR galaxies (LIRGs) and ULIRGs (e.g., Wilson et al. 2008; Sakamoto et al. 2014; Saito et al. 2017).

In IRAS 12112 and IRAS 22491, the continuum emission is dominated by the same nucleus that dominates the $\mathrm{CO}(2-1)$ emission (see Sect. 3.1.1). The fractions of the $248 \mathrm{GHz}$ continuum produced by these dominant nuclei are 90 and $>95 \%$, respectively, which are slightly higher than their contributions to the total $\mathrm{CO}(2-1)$ luminosity of their systems (80 and $90 \%$, respectively). In IRAS 14348 , the SW nucleus produces $60 \%$ 

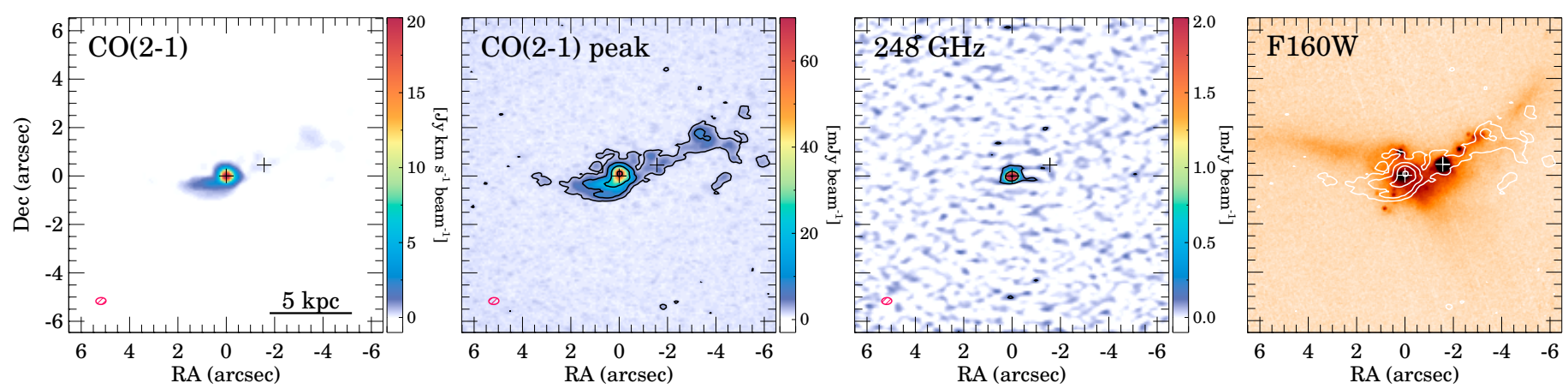

Fig. 3. Same as Fig. 1 but for IRAS 22491-1808.

Table 4. ALMA continuum models.

\begin{tabular}{|c|c|c|c|c|c|c|c|c|}
\hline Object & $\begin{array}{c}\text { Obs freq. }{ }^{a} \\
(\mathrm{GHz})\end{array}$ & $\begin{array}{l}\text { Rest freq. }{ }^{a} \\
(\mathrm{GHz})\end{array}$ & $\begin{array}{c}\text { Total flux } \\
\text { (mJy) }\end{array}$ & $\begin{array}{r}\text { Delta } \\
(\mathrm{mJy})\end{array}$ & $\begin{array}{c}\text { Gaussian }^{c} \\
(\mathrm{mJy})\end{array}$ & $\begin{array}{l}F W H M^{d} \\
(\operatorname{arcsec})\end{array}$ & $(\mathrm{pc})$ & $\begin{array}{l}\text { Non-thermal } \\
\text { fraction }^{e}\end{array}$ \\
\hline I12112 SW & 231.12 & 247.99 & $0.69 \pm 0.05$ & & $0.69 \pm 0.05$ & $0.19 \pm 0.02$ & 260 & 0.43 \\
\hline I12112 NE & & & $6.81 \pm 0.14$ & $4.60 \pm 0.10$ & $2.21 \pm 0.10$ & $0.35 \pm 0.02$ & 480 & 0.20 \\
\hline I14348 SW & 229.08 & 247.98 & $2.42 \pm 0.05$ & $\ldots$ & $2.42 \pm 0.05$ & $0.17 \pm 0.01$ & 260 & 0.21 \\
\hline I14348 NE & & & $1.63 \pm 0.05$ & $\ldots$ & $1.63 \pm 0.05$ & $0.17 \pm 0.01$ & 270 & 0.21 \\
\hline I22491 E & 229.64 & 247.50 & $5.16 \pm 0.11$ & $4.09 \pm 0.06$ & $1.07 \pm 0.10$ & $0.68 \pm 0.08$ & 1000 & 0.14 \\
\hline I22491 W & & & $<0.14^{f}$ & $\cdots$ & & $\cdots$ & $\cdots$ & $\cdots$ \\
\hline
\end{tabular}

Notes. The flux uncertainties are statistical uncertainties from the fit. The absolute flux calibration uncertainty is about $10 \%$. ${ }^{(a)}$ Observed and rest frame continuum frequencies. ${ }^{(b)}$ Total flux of the continuum model. ${ }^{(c)}$ Flux of the delta (unresolved) and Gaussian components of the models. ${ }^{(d)}$ Deconvolved FWHM of the Gaussian component. ${ }^{(e)}$ Non-thermal emission fraction at $248 \mathrm{GHz}$ estimated from the radio 1.49 and $8.44 \mathrm{GHz}$ fluxes (Condon et al. 1990, 1991; see Sect. 3.1.2). ${ }^{(f)} 3 \sigma$ flux upper limit for an unresolved source.

of the continuum emission and the NW produces the remaining $40 \%$. These fractions are similar to those of the $\mathrm{CO}(2-1)$ produced in each nucleus (65 and 35\%, respectively).

The $248 \mathrm{GHz}$ continuum emission is possibly produced by a combination of thermal dust continuum, free-free radio continuum, and synchrotron emission. The latter can be dominant at this frequency in the case of AGN, and, as discussed in Sect. 2.1, AGN emission is detected in the three ULIRGs. To determine the non-thermal contribution to the measured $248 \mathrm{GHz}$ fluxes, we use the available interferometric radio (1.49 and $8.44 \mathrm{GHz}$ ) observations for these systems (Condon et al. 1990, 1991). The position of the $248 \mathrm{GHz}$ continuum sources is compatible with the location of the 1.49 and $8.44 \mathrm{GHz}$ radio continuum emission within 0.15 ( $\sim$ half of the beam FWHM). Therefore, we assume that the radio and the $248 \mathrm{GHz}$ emissions are produced in the same regions. We also note that the western nucleus of IRAS 22491 is undetected as well at radio wavelengths. For the rest of the sources, we fit a power law to the 1.49 and $8.44 \mathrm{GHz}$ fluxes and obtain spectral indexes between 0.42 and 0.72 . Then, we use these spectral indexes to extrapolate the non-thermal emission at $248 \mathrm{GHz}$. On average, this represents $20 \%$ of the $248 \mathrm{GHz}$ emission for these ULIRGs (see Table 4), with a minimum (maximum) contribution of $14 \%$ (43\%). Therefore, most of the $248 \mathrm{GHz}$ emission is likely due to thermal dust emission and free-free radio continuum produced in the compact nuclear region.

\subsection{Molecular gas kinematics}

\subsubsection{Global kinematics}

In Fig. 4, we show the first and second moments of the $\mathrm{CO}(2-1)$ emission for each galaxy of the three ULIRG systems and indicate the outflow axis (dotted line) and the kinematic major axis of the nuclear disk (dashed line) defined in Sect. 3.2 (see Fig. 5). The first moment maps indicate a complex velocity field, although a rotating disk pattern is present in all the systems. The second moment maps show that the velocity dispersion maximum (120-170 $\left.\mathrm{km} \mathrm{s}^{-1}\right)$ is almost coincident with the location of the nucleus and that it is enhanced more or less along the molecular outflow axis (dotted line). The latter is expected since the high-velocity outflowing gas produces broad wings in the $\mathrm{CO}(2-1)$ line profile, which enhances the observed second moment.

\subsubsection{Nuclear disks and molecular outflows}

Similar to García-Burillo et al. (2015), we derive the centroid of the $\mathrm{CO}(2-1)$ emission in each velocity channel to study the nuclear gas kinematics and identify high-velocity gas decoupled from the rotating disks. The results are presented in Fig. 5. Thanks to the high signal-to-noise ratio $(\mathrm{S} / \mathrm{N})$ of the data, we are able to determine the centroid positions with statistical uncertainties $<10$ mas for most of the channels. The astrometric accuracy for the frequency and array configuration of these observations is $\sim 25$ mas for channels with a $S / N$ higher than 10 . For channels with a $\mathrm{S} / \mathrm{N}$ of approximately 3, this accuracy is reduced to $\sim 80$ mas $^{1}$. Therefore, the shifts of the centroid positions shown in Fig. 5 are expected to be real.

For all the objects, the low-velocity emission centroids follow a straight line. This is consistent with the emission from a rotating disk that is not completely resolved. The direction of this line traces the position angle (PA) of the kinematic major axis of the rotating disk. Therefore, we did a linear fit to these

1 See Sect. 10.5.2 of the ALMA Cycle 6 Technical Handbook. 

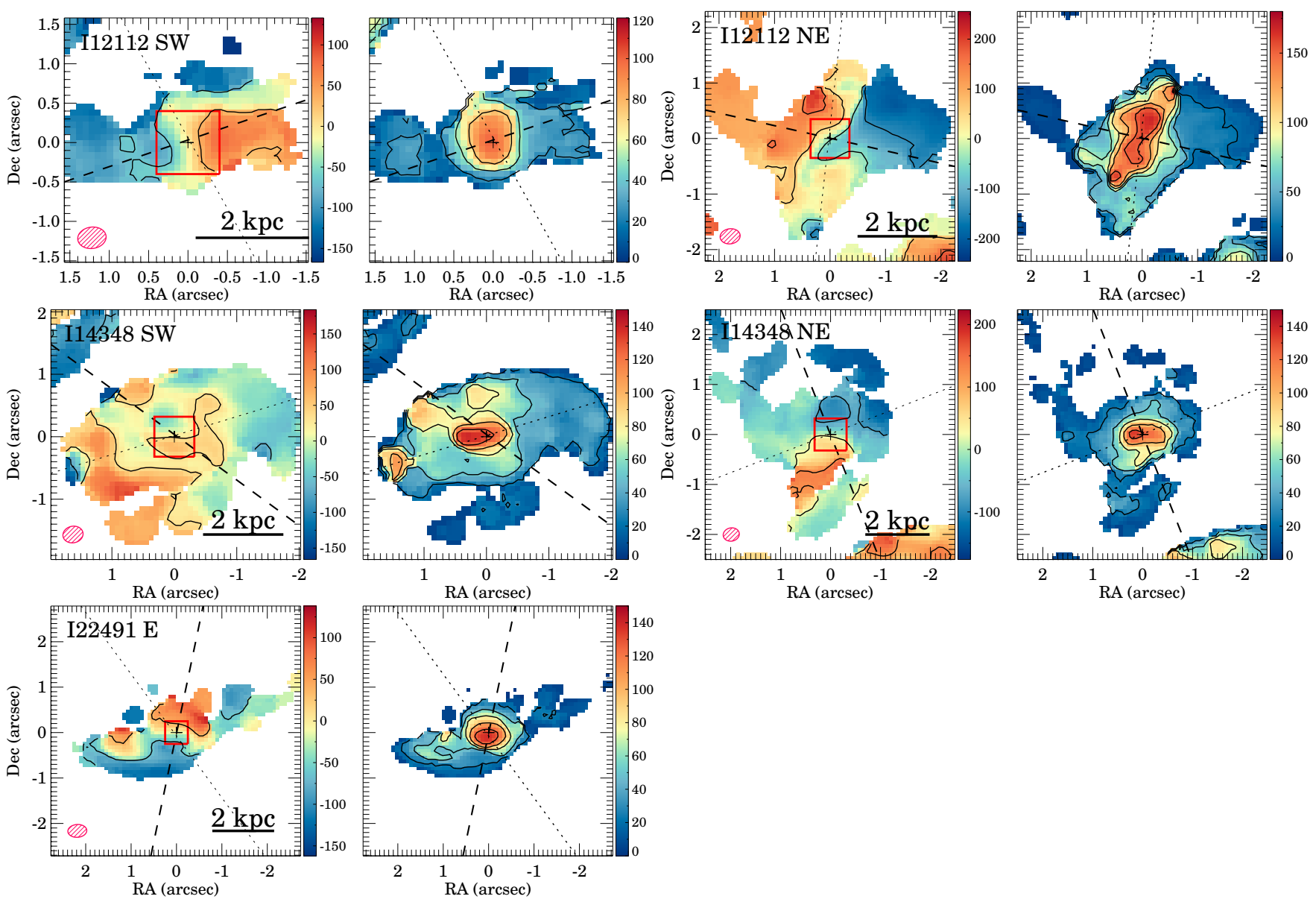

Fig. 4. For each galaxy of the ULIRG systems, the left-and right-hand panels show the first and second moments of the CO(2-1) emission, respectively. The spacings between the contour levels in the first and second moment maps are 100 and $25 \mathrm{~km} \mathrm{~s}^{-1}$, respectively. For the first moment maps, the velocities are relative to the systemic velocity (see Table 1). The red box in this panel indicates the field of view presented in Fig. 5 for each object. The dashed and dotted lines mark the kinematic major axis and the outflow axis, respectively, defined in Sect. 3.2 (see also Fig. 5). The black cross marks the position of the $248 \mathrm{GHz}$ continuum peak. The red hatched ellipse shows the beam FWHM and PA.

points and derived the disk PA (Table 5). Also, using these fits, we determined the systemic velocity as the velocity of the point along the major axis closest to the continuum peak (Table 1).

By contrast, the centroids of the high-velocity gas do not lie on the kinematic major axis and they cluster at two positions, one for the red-shifted and the other for the blue-shifted gas. These two positions are approximately symmetric with respect to the nucleus. This is strong evidence of the decoupling of the high-velocity gas from the global disk rotation and, as we discuss in Sect. 3.3, this is compatible with the expected gas distribution of a massive molecular outflow originating at the nucleus. Alternatively, if we assume a coplanar geometry for the highvelocity gas, these PA twists could be explained by a strong nuclear bar-like structure. However, because of the extremely high radial velocities implied by this geometry, up to $400 \mathrm{~km} \mathrm{~s}^{-1}$, in the following, we only discuss the out-of-plane outflow possibility.

The inclination of the disks is an important parameter to derive accurate outflow properties. It is commonly derived from the ratio between the photometric major and minor axes assuming that the galaxy is circular. However, in these merger systems, it is not obvious how to define these axes and also the circular morphology assumption might be incorrect. Therefore, we use an alternative approach based on the kinematic properties of the nuclear disks to estimate the inclination. First, we extract the rotation curve along the kinematic major axis, and fit an arctan model (e.g., Courteau 1997) to determine the curve semi-amplitude, $v$ (Fig. 6 and Table 5). Then, we measure the velocity dispersion, $\sigma$, of the nuclear region $(1-2 \mathrm{kpc})$ and calculate the observed dynamical ratio $v / \sigma$. García-Marín et al. (2006) and Bellocchi et al. (2013) measured the $v / \sigma$ ratios in a sample of 25 ULIRGs (34 individual galaxies) with $\mathrm{H} \alpha$ integral field spectroscopy. Assuming a mean inclination of $57^{\circ}$ ( $\sin i=0.79$; see Law et al. 2009), we can correct their $v$ values for inclination and determine an intrinsic $v / \sigma$ ratio of 1.5 \pm 0.6 for ULIRGs. Then, we compare the observed dynamical ratios in each galaxy with this intrinsic ratio to estimate their inclinations $\left(15-40^{\circ}\right)$.

\subsection{Properties of the molecular outflows}

\subsubsection{Observed properties: PA, size, flux, and velocity}

In the previous section, we presented the detection of highvelocity gas in five out of six ULIRG nuclei, which is compatible with the presence of massive molecular outflows. But depending on which side of the rotating disk is closest to us, this high-velocity gas can be interpreted as an inflow or as an outflow. To investigate this, we plot the morphological features (spiral arms or tidal tails) we identified in the $\mathrm{CO}(2-1)$ channel maps 

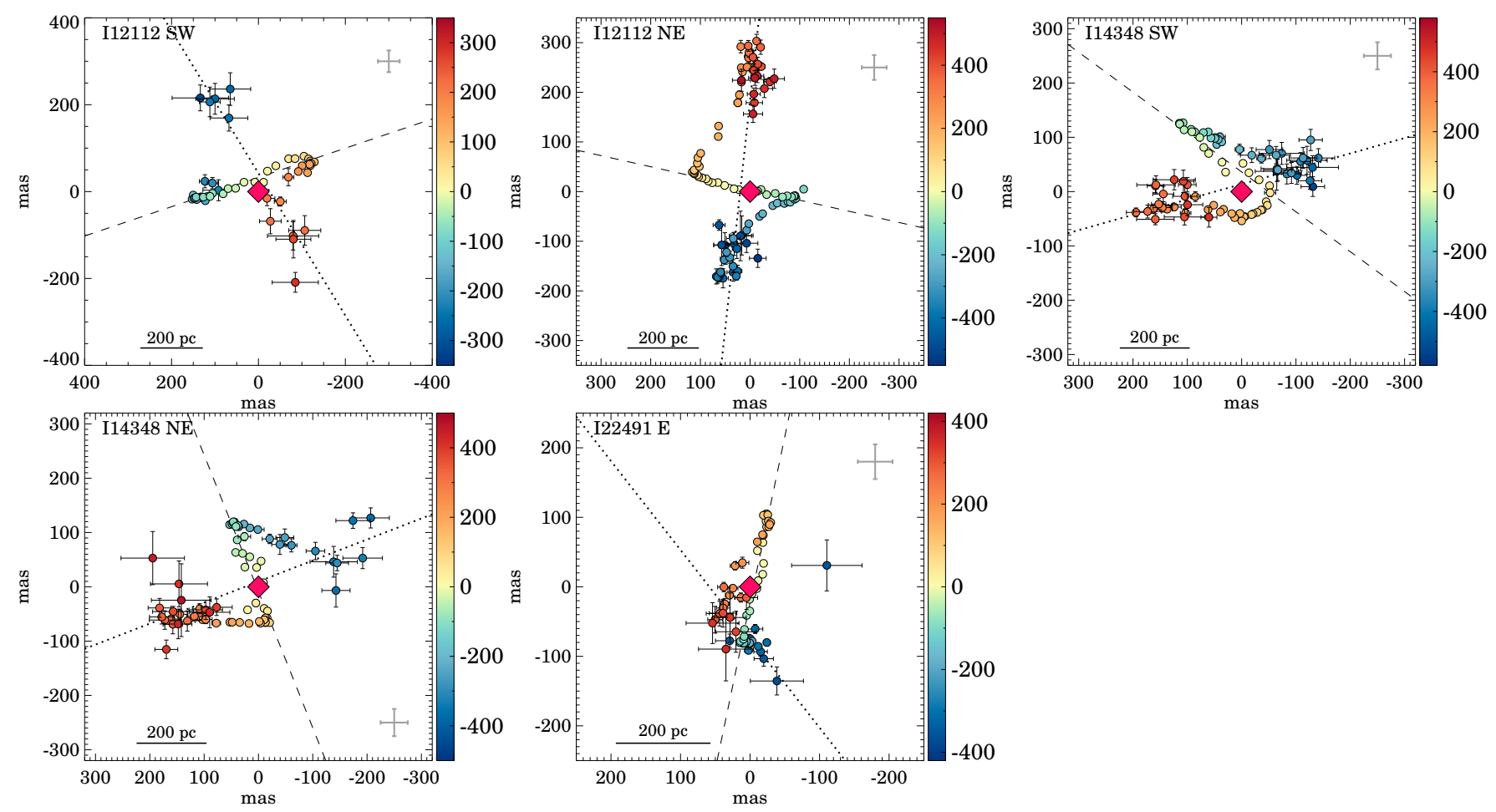

Fig. 5. Centroid of the $\mathrm{CO}(2-1)$ emission measured in each $\sim 10 \mathrm{~km} \mathrm{~s}^{-1}$ velocity channel. The color of the points indicates the $\mathrm{CO}(2-1)$ velocity with respect to the systemic velocity not corrected for inclination. The rose diamond marks the position of the $248 \mathrm{GHz}$ continuum peak. The dashed line is the linear fit to the low velocity gas and corresponds to the kinematic major axis of the rotating disk. The dotted line is the linear fit to the high-velocity red- and blue-shifted gas, which traces the projection of the outflow axis in the sky. The error bars in each point indicate the statistical uncertainty in the centroid position. The gray error bars represent the astrometric accuracy of these observations for channels with $S / N>10$ (see Sect. 3.2).

Table 5. Nuclear molecular emission.

\begin{tabular}{lccccccc}
\hline \hline Object & $\begin{array}{c}\mathrm{PA}^{a} \\
(\mathrm{deg})\end{array}$ & $\begin{array}{c}\mathrm{PA}_{\text {out }}^{b} \\
(\mathrm{deg})\end{array}$ & $\begin{array}{c}\mathrm{PA}^{-\mathrm{PA}_{\text {out }}{ }^{c}} \\
(\mathrm{deg})\end{array}$ & $\begin{array}{c}v^{d} \\
\left(\mathrm{~km} \mathrm{~s}^{-1}\right)\end{array}$ & $\begin{array}{c}\sigma^{e} \\
\left(\mathrm{~km} \mathrm{~s}^{-1}\right)\end{array}$ & $v / \sigma^{f}$ & $\begin{array}{c}i^{g} \\
(\mathrm{deg})\end{array}$ \\
\hline I12112 SW & $289 \pm 2$ & $213 \pm 10$ & $75 \pm 11$ & $81 \pm 6$ & $130 \pm 9$ & $0.62 \pm 0.09$ & $25 \pm 14$ \\
I12112 NE & $80 \pm 2$ & $353 \pm 5$ & $87 \pm 6$ & $120 \pm 8$ & $168 \pm 3$ & $0.71 \pm 0.06$ & $28 \pm 15$ \\
I14348 SW & $232 \pm 4$ & $107 \pm 8$ & $126 \pm 9$ & $60 \pm 10$ & $148 \pm 4$ & $0.40 \pm 0.09$ & $15 \pm 10$ \\
I14348 NE & $202 \pm 5$ & $112 \pm 7$ & $90 \pm 9$ & $120 \pm 40$ & $138 \pm 6$ & $0.89 \pm 0.30$ & $36 \pm 31$ \\
I22491 E & $348 \pm 2$ & $36 \pm 20$ & $133 \pm 20$ & $110 \pm 10$ & $122 \pm 5$ & $0.88 \pm 0.12$ & $36 \pm 23$ \\
\hline
\end{tabular}

Notes. ${ }^{(a, b)}$ Position angle (east of north) of the receding part of the kinematic major axis and the high-velocity outflowing gas, respectively (see Sect. 3.2 and Fig. 5). ${ }^{(c)}$ Difference between the position angles of the outflow and the kinematic major axis. ${ }^{(d)}$ Semi-amplitude of the observed $\mathrm{CO}(2-1)$ rotation curve. ${ }^{(e)}$ Second moment of the nuclear $\mathrm{CO}(2-1)$ emission profile (Figs. 7-11). ${ }^{(f)}$ Observed dynamical ratio. ${ }^{(g)}$ Disk inclination assuming an intrinsic dynamical ratio for local ULIRGs of $1.5 \pm 0.6$ (see sect. 3.2; García-Marín et al. 2006; Bellocchi et al. 2013).

(panel d of Figs. 7-11 and Appendix B). Then, in panel e of these figures, we plot the identified morphological features over the velocity fields and, assuming that these features are trailing, we can determine the near- and far-side of the rotating disk. For all the galaxies where the high-velocity emission is spatially resolved, the blue-shifted high-velocity emission appears on the far side of the disk and the red-shifted emission on the near side. This is a clear signature of outflowing gas.

In addition, we can measure the PA of these outflows by fitting the position of the red and blue centroid clusters with a straight line (Fig. 5 and Table 5). We calculated the difference between the PA of the high-velocity gas and that of the kinematic major axis of the disk (Table 5) and found values around $90^{\circ}$ for three cases. This PA difference is the expected value for an outflow perpendicular to the rotating disk. For IRAS 14348 SW, the PA difference is $\sim 126 \pm 8^{\circ}$, which suggests a different outflow orientation. Finally, the outflow PA of IRAS 22491 E seems to deviate from a perpendicular orientation although with less significance due to the large uncertainty $\left(\sim 120 \pm 20^{\circ}\right)$.

In panel a of Figs. 7-11, we show the spatial distribution of the high-velocity gas emission. This emission is spatially resolved, except for IRAS $22491 \mathrm{E}$, and reaches projected distances, $R_{\max }$, up to $0.4-1^{\prime \prime} .2(0.5-1.8 \mathrm{kpc}$; see Table 6$)$ from the nucleus. We note that these sizes are a factor of three to five larger than the sizes derived from the separation between the blue- and red-shifted emission centroids $\left(R_{\mathrm{c}}\right)$. In the following, we use the $R_{\mathrm{c}}$ as the outflow size because, as a flux-weighted estimate of the outflow size, it is a better estimate of the extent of the region where most of the outflowing molecular gas is located. On the other hand, $R_{\max }$ is dominated by the faint $\mathrm{CO}(2-1)$ emission 

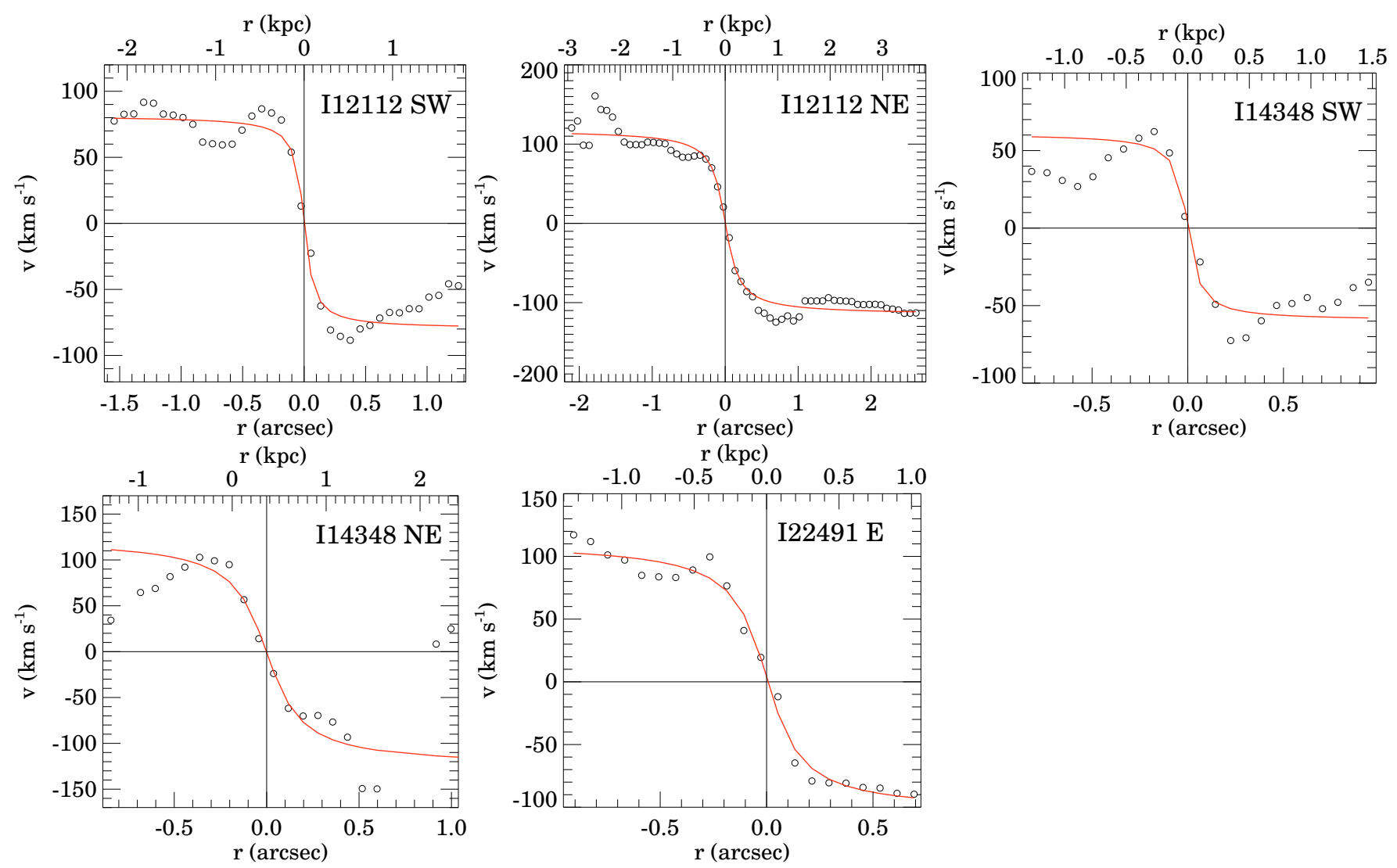

Fig. 6. Rotation curves of the ULIRGs extracted along the kinematic major axis. The red line shows the best fit arctan model to the data. The fit results are presented in Table 5.
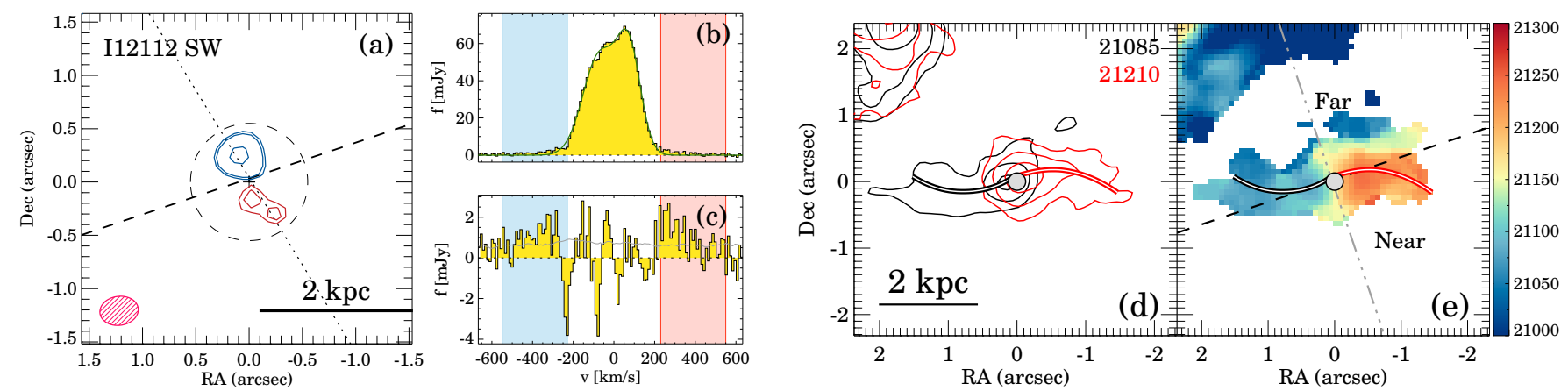

Fig. 7. Blue and red contours in panel $a$ represent the integrated blue- and red-shifted high-velocity $\mathrm{CO}(2-1)$ emission, respectively. The specific velocity range is listed in Table 6 . The lowest contour corresponds to the $3 \sigma$ level. The next contour levels are $(0.5,0.9) \times$ the peak of the high-velocity emission when these are above the $3 \sigma$ level. For I12112 SW, $\sigma=30 \mathrm{mJy} \mathrm{km} \mathrm{s}^{-1}$ beam $^{-1}$ and the red and blue peaks are at 110 and $240 \mathrm{mJy} \mathrm{km} \mathrm{s}^{-1}$ beam $^{-1}$, respectively. The dotted and the dashed lines are the outflow axis and the kinematic major axis, respectively (see Table 5). The red hatched ellipse represents the beam FWHM and PA. The dashed circle indicates the region from which the nuclear spectrum was extracted. Panel $b$ : nuclear spectrum in yellow and the best-fit model in gray. Panel $c$ : difference between the observed spectrum and the best-fit model. The shaded blue and red velocity ranges in these panels correspond to the velocity ranges used for the contours of panel a. The gray line in panel $c$ marks the $3 \sigma$ noise level per channel. Panel $d$ : $\mathbf{C O}(2-1)$ emission at the velocities indicated by the numbers at the top-right corner of the panel as black and red contours, respectively. The black and red double lines trace the morphological features (spiral arms, tidal tails) observed at those velocities. Panel $e$ : $\mathrm{CO}(2-1)$ mean velocity field (same as in Fig. 4). The black dashed line is the kinematic major axis and the gray dot-dashed line is the kinematic minor axis. The far and near sides of the rotating disk are indicated, assuming that the observed morphological features are trailing.

at larger radii and it is also likely dependent on the sensitivity of the observations.

The outflows are clearly spatially resolved $\left(2 \times R_{\max }>\right.$ $F W H M$ of the beam). However, the angular resolution is not high enough to allow us to measure the outflow properties as a function of radius. For this reason, we only consider the integrated outflow emission and measure a total outflow flux. To do so, we extracted the spectrum of the regions where high-velocity gas is detected (panel b of Figs. 7-11). We fit a two-Gaussian model to the $\mathrm{CO}(2-1)$ line profile. This model reproduces well the core of the observed line profile. The residual blue and red wings (i.e., the outflow emission) are shown in panel c of Figs. 7-11 for each galaxy. From these spectra, we also estimate the flux-weighted average velocity of the outflowing gas $\left(320-460 \mathrm{~km} \mathrm{~s}^{-1}\right)$ and 

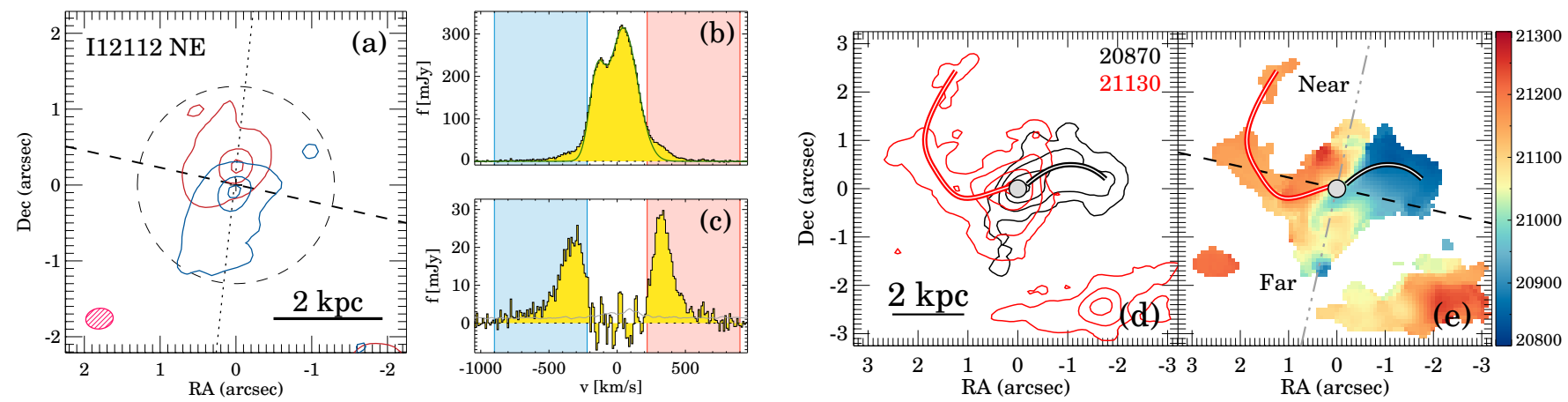

Fig. 8. Same as Fig. 7 but for I12112 NE. In panel $a, \sigma=45 \mathrm{mJy} \mathrm{km} \mathrm{s}^{-1}$ beam $^{-1}$ and the red and blue peaks are at 2.4 and $2.9 \mathrm{Jy} \mathrm{km} \mathrm{s}^{-1}$ beam ${ }^{-1}$, respectively.
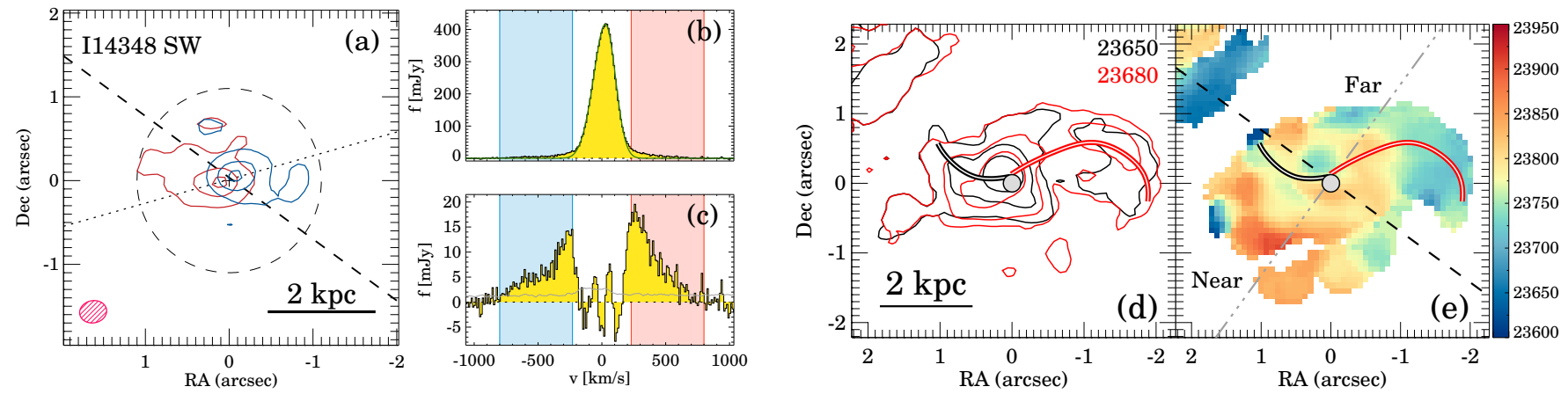

Fig. 9. Same as Fig. 7 but for I14348 SW. In panel a, $\sigma=46 \mathrm{mJy} \mathrm{km} \mathrm{s}^{-1}$ beam $^{-1}$ and the red and blue peaks are at 1.4 and $1.0 \mathrm{Jy} \mathrm{km} \mathrm{s}^{-1}$ beam ${ }^{-1}$, respectively.
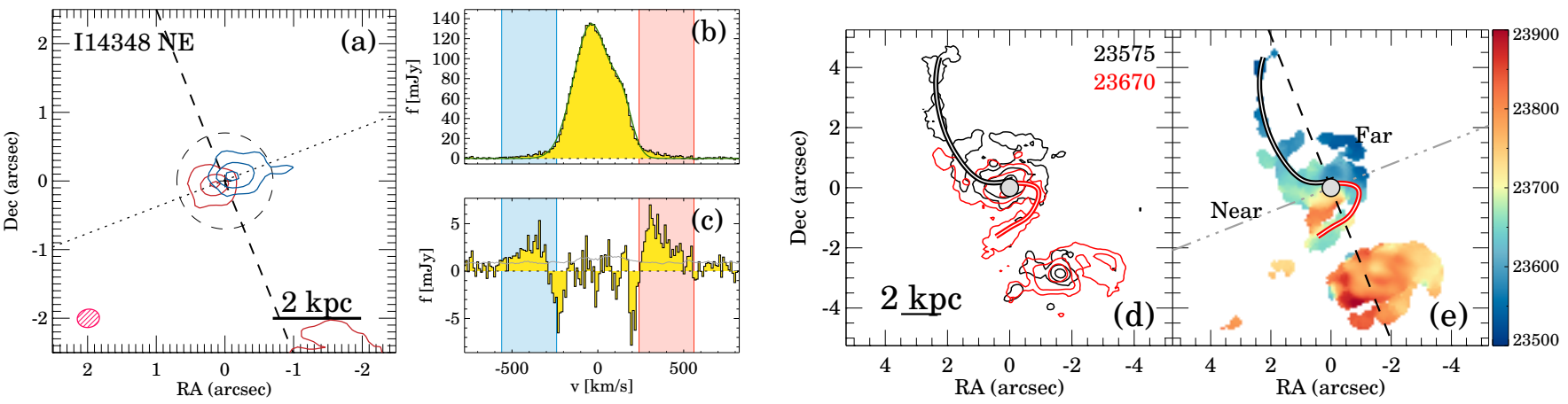

Fig. 10. Same as Fig. 7 but for I14348 NE. In panel $a, \sigma=32 \mathrm{mJy} \mathrm{km} \mathrm{s}^{-1}$ beam ${ }^{-1}$ and the red and blue peaks are at 0.74 and $0.56 \mathrm{Jy} \mathrm{km} \mathrm{s}^{-1}$ beam ${ }^{-1}$, respectively.

the maximum velocity at which we detect $\mathrm{CO}(2-1)$ emission $\left(400-800 \mathrm{~km} \mathrm{~s}^{-1}\right)$. The total $\mathrm{CO}(2-1)$ flux, as well as the flux in the high-velocity wings, are presented in Table 6.

\subsubsection{Derived properties}

In Table 7, we present the derived properties for these outflows based on the observations and assuming that they are perpendicular to the rotating disk. The latter is consistent with the $\sim 90^{\circ} \mathrm{PA}$ difference between the kinematic major axis and the outflow axis measured in three of the galaxies. For the other two cases (PA difference $\sim 120^{\circ}$ ), this assumption might introduce a factor of approximately two uncertainty into the derived outflow rates.

To convert the $\mathrm{CO}(2-1)$ fluxes into molecular masses, we assume a ULIRG-like $\alpha_{\mathrm{CO}}$ conversion factor of $0.78 M_{\odot}\left(\mathrm{K} \mathrm{km} \mathrm{s}^{-1} \mathrm{pc}^{-2}\right)^{-1}$ and a ratio between the $\mathrm{CO}(2-1)$ and $\mathrm{CO}(1-0)$ transitions, $r_{21}$, of 0.91 (Bolatto et al. 2013). The outflow velocity is corrected for the inclination by dividing by $\cos i$, where $i$ is the disk inclination (Table 5). Similarly, the outflow radius is corrected by dividing by $\sin i$. The average corrected outflow velocity is $\sim 440 \mathrm{~km} \mathrm{~s}^{-1}$ and the average deprojected radius is $\sim 700 \mathrm{pc}$.

Using these quantities, we calculate the dynamical time as $t_{\text {dyn }}=R_{\text {out }} / v_{\text {out }}$ (about $\sim 1$ Myr for these outflows). Then, we estimate the outflow rate using $\dot{M}_{\text {out }}=M_{\text {out }} / t_{\text {dyn }}$. We find $\dot{M}_{\text {out }}$ values between $\sim 12$ and $\sim 400 M_{\odot} \mathrm{yr}^{-1}$. From these estimates, we can derive the depletion time $\left(t_{\mathrm{dep}}\right)$, outflow momentum rate $\left(\dot{P}_{\text {out }}\right)$, and the outflow kinetic luminosity $\left(L_{\text {out }}\right.$; see, e.g., García-Burillo et al. 2015).

The uncertainties on the outflow rate, and the quantities derived from it, are dominated by the uncertainty in the value of the $\alpha_{\mathrm{CO}}$ conversion factor, which is not well established for the outflowing gas (e.g., Aalto et al. 2015) and the outflow geometry (inclination). For the conversion factor, we assume a 

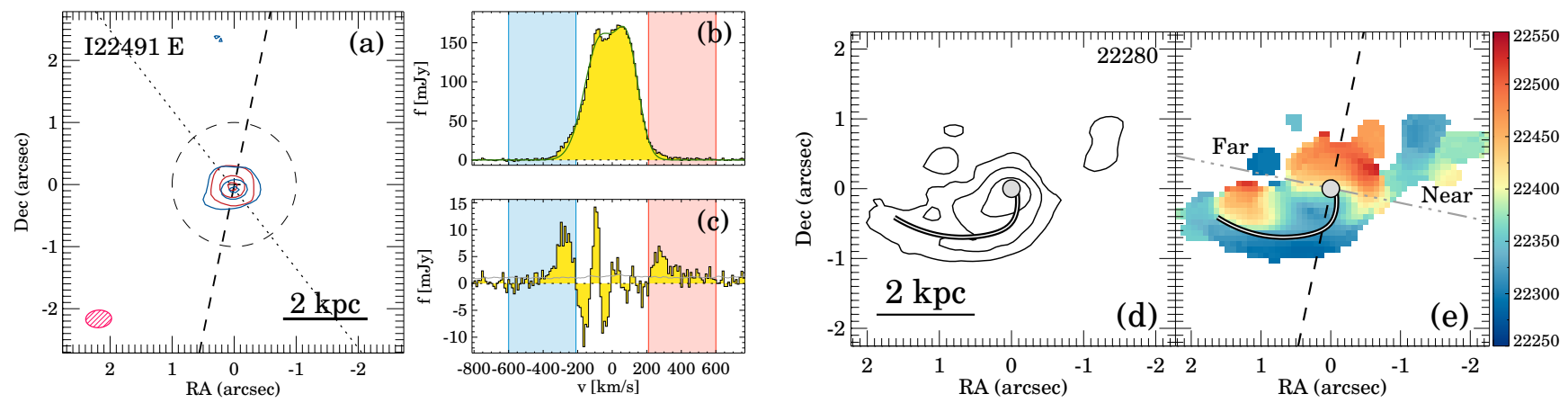

Fig. 11. Same as Fig. 7 but for I22491 E. In panel $a, \sigma=43 \mathrm{mJy} \mathrm{km} \mathrm{s}^{-1}$ beam $^{-1}$ and the red and blue peaks are at 1.1 and $2.2 \mathrm{Jy} \mathrm{km} \mathrm{s}^{-1}$ beam $^{-1}$, respectively.

Table 6. Nuclear $\mathrm{CO}(2-1)$ emission and observed outflow properties.

\begin{tabular}{|c|c|c|c|c|c|c|c|c|c|}
\hline \multirow[t]{2}{*}{ Object } & \multirow{2}{*}{$\begin{array}{c}r^{a} \\
(\operatorname{arcsec}[\mathrm{kpc}])\end{array}$} & \multirow{2}{*}{$\begin{array}{l}v \text { range }^{b} \\
\left(\mathrm{~km} \mathrm{~s}^{-1}\right)\end{array}$} & \multicolumn{3}{|c|}{$S_{\mathrm{CO}}$} & \multirow{2}{*}{$\begin{array}{c}\left|v_{\text {high }}\right|^{e} \\
\left(\mathrm{~km} \mathrm{~s}^{-1}\right) \\
\end{array}$} & \multirow{2}{*}{$\begin{array}{c}v_{\max }^{f} \\
\left(\mathrm{~km} \mathrm{~s}^{-1}\right)\end{array}$} & \multirow{2}{*}{$\begin{array}{c}R_{\mathrm{c}}{ }^{g} \\
(\operatorname{arcsec}) \\
\end{array}$} & \multirow{2}{*}{$\begin{array}{l}R_{\max }^{h} \\
(\operatorname{arcsec})\end{array}$} \\
\hline & & & $\begin{array}{c}\text { Total }^{c} \\
\left(\mathrm{Jy} \mathrm{km} \mathrm{s}^{-1}\right)\end{array}$ & $\begin{array}{c}\text { Blue-shifted } \\
\left(\mathrm{Jy} \mathrm{km} \mathrm{s}^{-1}\right)\end{array}$ & $\begin{array}{l}\text { Red-shifted } \\
\left(\mathrm{Jy} \mathrm{km} \mathrm{s}^{-1}\right)\end{array}$ & & & & \\
\hline I12112 SW & $0.55[0.8]$ & {$[230,550]$} & $19.5 \pm 0.1$ & $0.25 \pm 0.04$ & $0.32 \pm 0.04$ & $324 \pm 12$ & $470 \pm 20$ & $0.24 \pm 0.03$ & $0.55 \pm 0.05$ \\
\hline I12112 NE & $1.3[1.8]$ & {$[220,900]$} & $115.9 \pm 0.2$ & $5.4 \pm 0.1$ & $5.3 \pm 0.1$ & $465 \pm 30$ & $800 \pm 90$ & $0.24 \pm 0.03$ & $1.15 \pm 0.30$ \\
\hline I14348 SW & $1.1[1.7]$ & {$[230,800]$} & $97.7 \pm 0.2$ & $3.5 \pm 0.1$ & $4.1 \pm 0.1$ & $419 \pm 38$ & $740 \pm 30$ & $0.18 \pm 0.03$ & $1.05 \pm 0.05$ \\
\hline I14348 NE & $0.7[1.1]$ & {$[240,560]$} & $42.1 \pm 0.1$ & $0.48 \pm 0.05$ & $1.0 \pm 0.1$ & $373 \pm 5$ & $520 \pm 110$ & $0.22 \pm 0.03$ & $0.60 \pm 0.05$ \\
\hline I22491 E & $1.0[1.5]$ & {$[210,600]$} & $57.4 \pm 0.1$ & $1.1 \pm 0.1$ & $0.9 \pm 0.1$ & $325 \pm 33$ & $400 \pm 110$ & $0.10 \pm 0.01$ & $0.35 \pm 0.05$ \\
\hline
\end{tabular}

Notes. ${ }^{(a)}$ Radius of the aperture used to measure the $\mathrm{CO}(2-1)$ emission in arcsec and kpc. ${ }^{(b)}$ Absolute value of the velocity range considered to measure the blue- and red-shifted wings of the $\mathrm{CO}(2-1)$ profile with respect to the systemic velocity. ${ }^{(c)} \mathrm{Total} \mathrm{CO}(2-1)$ flux measured within an aperture of radius $r$ centered on the object nucleus. ${ }^{(d)}$ Blue- and red-shifted high-velocity $\mathrm{CO}(2-1)$ emission measured in the indicated velocity range after subtracting the best-fit two-Gaussian model (see Sect. 3.3.1 and Figs. 7-11). ${ }^{(e)}$ Absolute value of the intensity weighted average velocity of the high-velocity gas with respect to the systemic velocity. ${ }^{(f)}$ Maximum velocity at which $\mathrm{CO}(2-1)$ emission is detected at more than $3 \sigma .{ }^{(g)} \mathrm{Half}$ of the maximum separation between the centroids of the blue- and red-shifted high-velocity emission with the same $\left|v-v_{\text {sys }}\right|\left(\right.$ see Fig. 5). ${ }^{(h)}$ Largest radius at which high-velocity $\mathrm{CO}(2-1)$ emission is detected.

Table 7. Derived molecular outflow properties.

\begin{tabular}{|c|c|c|c|c|c|c|c|c|c|c|c|}
\hline Object & $\begin{array}{c}M_{\text {out }^{a}} \\
\left(10^{8} M_{\odot}\right) \\
\end{array}$ & $\begin{array}{c}M_{\mathrm{tot}}{ }^{b} \\
\left(10^{9} M_{\odot}\right)\end{array}$ & $\begin{array}{c}v_{\text {out }^{c}} \\
\left(\mathrm{~km} \mathrm{~s}^{-1}\right)\end{array}$ & $\begin{array}{c}v_{\max }{ }^{d} \\
\left(\mathrm{~km} \mathrm{~s}^{-1}\right)\end{array}$ & $\begin{array}{r}R_{\text {out }}^{e} \\
(\mathrm{pc})\end{array}$ & $\begin{array}{r}R_{\max }^{f} \\
(\mathrm{kpc})\end{array}$ & $\begin{array}{c}\log t_{\mathrm{dyn}}{ }^{g} \\
(\mathrm{yr})\end{array}$ & $\begin{array}{l}\log \dot{M}_{\text {out }}{ }^{h} \\
\left(M_{\odot} \mathrm{yr}^{-1}\right)\end{array}$ & $\begin{array}{c}\log t_{\mathrm{dep}^{i}} \\
(\mathrm{yr})\end{array}$ & $\begin{array}{c}\log \dot{P}_{\text {out }}{ }^{j} \\
\left(\mathrm{~g} \mathrm{~cm} \mathrm{~s}^{-2}\right)\end{array}$ & $\begin{array}{c}\log L_{\text {out }}{ }^{k} \\
\left(\mathrm{erg} \mathrm{cm}^{-2} \mathrm{~s}\right)\end{array}$ \\
\hline I12112 SW & 0.31 & 1.0 & 360 & 520 & 810 & 1.8 & 6.3 & 1.1 & 7.9 & 34.49 & 41.74 \\
\hline I12112 NE & 5.7 & 6.1 & 530 & 910 & 710 & 3.4 & 6.1 & 2.6 & 7.2 & 36.13 & 43.55 \\
\hline I14348 SW & 5.2 & 6.7 & 430 & 770 & 1000 & 6.0 & 6.4 & 2.4 & 7.5 & 35.79 & 43.13 \\
\hline I14348 NE & 1.0 & 2.9 & 460 & 650 & 590 & 1.5 & 6.1 & 1.9 & 7.5 & 35.38 & 42.75 \\
\hline I22491 E & 1.2 & 3.5 & 400 & 500 & 250 & 0.9 & 5.8 & 2.3 & 7.2 & 35.72 & 43.02 \\
\hline
\end{tabular}

Notes. ${ }^{(a, b)}$ Outflow and integrated molecular gas masses, respectively, assuming a ULIRG-like conversion factor $\alpha_{\mathrm{CO}}$ of $0.78 M_{\odot}\left(\mathrm{K} \mathrm{km} \mathrm{s}^{-1} \mathrm{pc}^{-2}\right)^{-1}$ and $r_{21}$ ratio of 0.91 (Bolatto et al. 2013). ${ }^{(c)}$ Inclination corrected outflow velocity $\mid v_{\text {high }} / \cos i$ (see Table 6). ${ }^{(d)}$ Inclination corrected maximum outflow velocity $\left|v_{\max }\right| / \cos i$ (see Table 6). ${ }^{(e)}$ Inclination corrected outflow radius range estimated from $R_{\mathrm{c}} / \sin i$ (see Table 6). ${ }^{(f)}$ Inclination corrected outflow maximum radius derived using $R_{\max } / \sin i$ (see Table 6). ${ }^{(g)}$ Outflow dynamical time $t_{\text {dyn }}=R_{\text {out }} / v_{\text {out }}$ (see Table 6). ${ }^{(h)}$ Outflow rate $\dot{M}_{\text {out }}=v_{\text {out }} \times M_{\text {out }} / R_{\text {out }}$. The uncertainty is $\sim 0.4 \mathrm{dex}$ (see Sect. 3.3.2). ${ }^{(i)}$ Depletion time $t_{\text {dep }}=M_{\text {tot }} / \dot{M}_{\text {out }}{ }^{(j)}$ Outflow momentum rate $\dot{P}_{\text {out }}=\dot{M}_{\text {out }} \times v_{\text {out }}$. ${ }^{(k)}$ Outflow kinetic luminosity $L_{\text {out }}=1 / 2 \times \dot{M}_{\text {out }} \times v_{\text {out }}^{2}$.

ULIRG-like value $\left(\alpha_{\mathrm{CO}}=0.78 M_{\odot}\left(\mathrm{K} \mathrm{km} \mathrm{s}^{-1} \mathrm{pc}^{-2}\right)^{-1}\right)$, although depending on the gas conditions, this factor can vary within 0.3 dex (e.g., Papadopoulos et al. 2012; Bolatto et al. 2013). Therefore, from the uncertainty in the inclination and the conversion factor, we assume a 0.4 dex uncertainty for these values.

Finally, in Table 8, we estimate the fraction of the outflowing gas that would escape the gravitational potential of these galaxies. We use the escape velocities at $2 \mathrm{kpc}$ calculated by Emonts et al. (2017) for these systems that range from $\sim 400$ to $600 \mathrm{~km} \mathrm{~s}^{-1}$. We integrate the $\mathrm{CO}(2-1)$ emission with velocities higher than these escape ones (taking into account the inclination of the outflows) and obtain that $15-30 \%$ of the high-velocity gas will escape to the intergalactic medium. The escape outflow rates are $3-120 M_{\odot} \mathrm{yr}^{-1}$. However, these escape rates can be lower if the velocity of the outflowing gas is decreased due to dynamical friction.

\subsection{Nuclear SFR}

Measuring the SFR in these ULIRGs is important to evaluate the impact of the molecular outflows. Most of the outflowing molecular gas is concentrated in the central $1-2 \mathrm{kpc}$, so to determine the local effect of the outflows, we must compare them with the nuclear SFR. However, the nuclei of local ULIRGs are extremely obscured regions (e.g., García-Marín et al. 2009a; Piqueras López et al. 2013) and estimating their SFR is not straightforward. In this section, we use two approaches to measure the nuclear SFR using the IR luminosity and the 
Table 8. Escape outflow.

\begin{tabular}{|c|c|c|c|c|c|}
\hline Object & $\begin{array}{c}v_{\mathrm{esc}^{a}} \\
\left(\mathrm{~km} \mathrm{~s}^{-1}\right)\end{array}$ & $\begin{array}{c}v_{\text {range }}{ }^{b} \\
\left(\mathrm{~km} \mathrm{~s}^{-1}\right)\end{array}$ & $\begin{array}{c}S_{\mathrm{CO}}^{\operatorname{esc} c} \\
\left(\mathrm{Jy} \mathrm{km} \mathrm{s}^{-1}\right)\end{array}$ & $S_{\mathrm{CO}}^{\mathrm{esc}} / S_{\mathrm{CO}}^{\text {out } d}$ & $\begin{array}{l}\log \dot{M}_{\mathrm{esc}}{ }^{e} \\
\left(M_{\odot} \mathrm{yr}^{-1}\right)\end{array}$ \\
\hline I12112 SW & 465 & {$[425,550]$} & $0.14 \pm 0.03$ & 0.24 & 0.49 \\
\hline I12112 NE & 465 & {$[414,900]$} & $3.0 \pm 0.1$ & 0.28 & 2.1 \\
\hline I14348 SW & 590 & {$[570,800]$} & $1.2 \pm 0.1$ & 0.16 & 1.6 \\
\hline I14348 NE & 590 & {$[459,560]$} & $0.28 \pm 0.04$ & 0.18 & 1.2 \\
\hline I22491 E & 400 & {$[319,600]$} & $0.7 \pm 0.1$ & 0.34 & 1.8 \\
\hline
\end{tabular}

Notes. ${ }^{(a)}$ Escape velocity at $2 \mathrm{kpc}$ (see Emonts et al. 2017). ${ }^{(b)}$ Observed velocity range used to measure the molecular gas with $v>v_{\mathrm{esc}}$ taking into account the inclination of the object. ${ }^{(c)} \mathrm{CO}(2-1)$ emission with $v>v_{\text {esc }}{ }^{\left({ }^{d}\right)}$ Ratio between the $\mathrm{CO}(2-1)$ emission from molecular gas with $v>v_{\text {esc }}$ and the total outflowing gas from Table $6 .{ }^{(e)}$ Molecular gas escape rate.
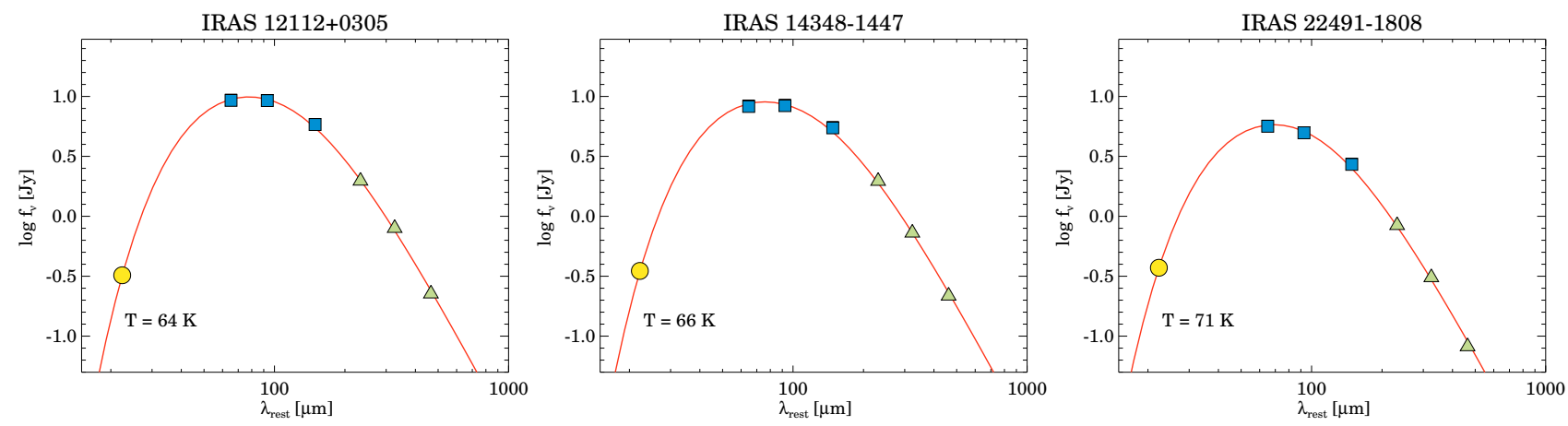

Fig. 12. Mid- and far-IR spectral energy distribution of the three ULIRGs. The yellow circle corresponds to the Spitzer/MIPS $24 \mu \mathrm{m}$ flux, the blue squares to the Herschel/PACS 60, 100, and $160 \mu \mathrm{m}$ fluxes, and the green triangles to the Herschel/SPIRE 250, 350, and 500 $\mu \mathrm{m}$ fluxes. The solid red line is the best fit to the data using a single temperature gray body.

$248 \mathrm{GHz}$ continuum and the radio continuum, which should not be heavily affected by extinction.

\subsubsection{IR luminosity}

The total IR luminosity $\left(L_{\mathrm{IR}}\right)$ is a good tracer of the SF in dusty environments such as the nuclei of ULIRGs (e.g., Kennicutt \& Evans 2012). However, there are no far-IR observations with the two nuclei of the systems spatially resolved. For this reason, we first derived the integrated $L_{\mathrm{IR}}$ of each system. We fit a single temperature gray body model to the $24-500 \mu \mathrm{m}$ fluxes from Spitzer and Herschel (Piqueras López et al. 2016; Chu et al. 2017) following Pereira-Santaella et al. (2016). The resulting $L_{\mathrm{IR}}$ are $\sim 0.2$ dex lower than those derived using the IRAS fluxes, but we consider these new $L_{\mathrm{IR}}$ more accurate since we are using more data points that cover a wider wavelength range to fit the IR emission (seven points between 24 and $500 \mu \mathrm{m}$ vs. four points between 12 and $100 \mu \mathrm{m}$ ) and also we avoid flux contamination from unrelated sources thanks to the higher angular resolution of the new data $\left(6^{\prime \prime}-35^{\prime \prime}\right.$ vs. $\left.0.5-2^{\prime}\right)$. The $L_{\mathrm{IR}}$ are listed in Table 1 and the best fits are shown in Fig. 12 .

Then, we assign a fraction of $L_{\mathrm{IR}}$ due to the star formation (i.e., after subtracting the AGN luminosity from Table 1) to each nucleus, which is proportional to their contribution to the total thermal continuum at $248 \mathrm{GHz}$ (dust emission plus free-free radio continuum; see Table 4) of the system. By doing this, we assume that all the $L_{\mathrm{IR}}$ is produced in the central $300-1000 \mathrm{pc}$, which is consistent with the compact distribution of the molecular gas around the nuclei (Sect. 3.1.1), and that the $248 \mathrm{GHz}$ continuum scales with the $L_{\mathrm{IR}}$. The latter is true for the free-free radio continuum contribution at this frequency, which is proportional to the ionizing flux and, therefore, to the SFR and
$L_{\mathrm{IR}}$. The dust emission at $248 \mathrm{GHz}$ depends on the average dust temperature of each nucleus $\left(f_{v} / L \propto T^{-3}\right.$ in the Rayleigh-Jeans tail of the black body). However, given the similar temperatures we obtained for the integrated emission $(T \sim 65-70 \mathrm{~K}$; see Fig. 12), our assumption seems to be reasonable. Finally, we converted these nuclear IR luminosities into SFR using the Kennicutt \& Evans (2012) calibration (see Table 9). The SFRs range from 13 to $180 M_{\odot} \mathrm{yr}^{-1}$.

\subsubsection{Radio continuum}

We also estimated the SFR from the non-thermal radio continuum observations of these galaxies (see Sect. 3.1.2). Using the observed 1.49 and $8.44 \mathrm{GHz}$ fluxes and the derived spectral indexes, we estimated the rest-frame $1.4 \mathrm{GHz}$ continuum and applied the Murphy et al. (2011) SFR calibration. Here, we ignore any contribution from an AGN to the radio emission. These objects seem to be dominated by SF and just have a small AGN contribution, but the radio emission of these AGN is uncertain.

The obtained radio SFR are listed in Table 9. These values are comparable to those obtained from the IR luminosity. The average difference between the two estimates is 0.2 dex with a maximum of 0.4 dex. Therefore, the two methods provide compatible SFR values and, in the following, we adopt the SFR(IR) with a 0.2 dex uncertainty.

\section{Discussion}

\subsection{Outflow energy source}

In the left panel of Fig. 13, we show the relation between the outflow rate and the nuclear SFR (i.e., the mass loading factor $\eta$ ). 
Table 9. Outflows and nuclear SFR.

\begin{tabular}{|c|c|c|c|c|c|}
\hline Object & $\begin{array}{c}\log \mathrm{SFR}(\mathrm{IR})^{a} \\
\left(M_{\odot} \mathrm{yr}^{-1}\right)\end{array}$ & $\begin{array}{c}\log \operatorname{SFR}(1.4 \mathrm{GHz})^{b} \\
\left(M_{\odot} \mathrm{yr}^{-1}\right)\end{array}$ & $\log \eta^{c}$ & $\log \frac{\dot{P}_{\text {out }}{ }^{d}{ }_{\mathrm{SNe}}}{d}$ & $\log \frac{L_{\text {out }}}{L_{\mathrm{SNe}}} e$ \\
\hline I12112 SW & 1.12 & 1.57 & -0.02 & -0.65 & -1.4 \\
\hline I12112 NE & 2.26 & 2.21 & 0.34 & -0.12 & -0.67 \\
\hline I14348 SW & 2.14 & 2.36 & 0.26 & -0.28 & -0.91 \\
\hline I14348 NE & 1.97 & 2.12 & -0.07 & -0.58 & -1.2 \\
\hline I22491 E & 2.04 & 1.76 & 0.26 & -0.32 & -0.99 \\
\hline
\end{tabular}

Notes. ${ }^{(a)}$ SFR derived from the IR luminosity assigned to each nucleus (see Sect. 3.4.1) using the calibration of Kennicutt \& Evans (2012). This is the adopted SFR for these nuclei. ${ }^{(b)}$ SFR derived from the non-thermal radio continuum using the Murphy et al. (2011) SFR calibration (see Sect. 3.4.2). ${ }^{(c)}$ Logarithm of the mass loading factor $\eta=\dot{M}_{\text {out }} / \mathrm{SFR}(\mathrm{IR}){ }^{(d)}$ Ratio between the outflow momentum rate and the momentum injected by supernova explosions. We assume that the $P_{\mathrm{SN}}$ per SN is $1.3 \times 10^{5} M_{\odot} \mathrm{km} \mathrm{s}^{-1} \times\left(n_{0} / 100 \mathrm{~cm}^{-3}\right)^{-0.17}\left(\right.$ Kim \& Ostriker 2015) using $n_{0}=100 \mathrm{~cm}^{-3}$ and that the SN rate, $v_{\mathrm{SN}}\left(\mathrm{yr}^{-1}\right)$, is $0.012 \times \operatorname{SFR}(\mathrm{IR})\left(M_{\odot} \mathrm{yr}^{-1}\right)$ for the adopted IMF (Leitherer et al. 1999). ${ }^{(e)}$ Ratio between the total kinetic luminosity of the molecular outflow and that injected by supernova explosions $\left(L_{\mathrm{SNe}}\left(\mathrm{erg} \mathrm{s}^{-1}\right)=9 \times 10^{41} \mathrm{SFR}(\mathrm{IR})\left(M_{\odot} \mathrm{yr}^{-1}\right)\right.$; Leitherer et al. 1999 , adapted for a Kroupa 2001 IMF).
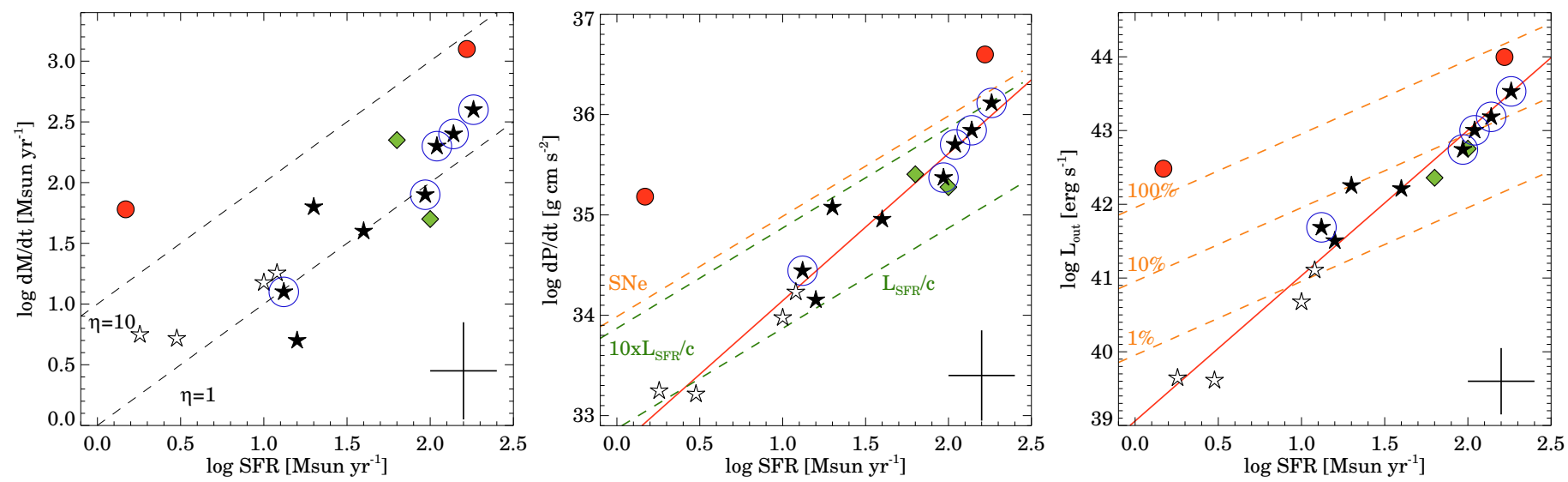

Fig. 13. Mass outflow rate vs. nuclear SFR (left panel), outflow momentum rate vs. nuclear SFR (middle panel), and outflow kinetic luminosity vs. nuclear SFR (right panel). Red circles indicate nuclei with outflows launched by an AGN, green diamonds are objects hosting an AGN with molecular outflows of uncertain SF/AGN origin, and stars represent star-formation-dominated nuclei. The blue circles mark the ULIRGs presented in this work. The white stars are the lower luminosity starburts compiled by Cicone et al. (2014). The remaining points correspond to local U/ LIRGs from the literature: NGC 1614 and IRAS 17208-0014 (García-Burillo et al. 2015; Pereira-Santaella et al. 2015; Piqueras López et al. 2016); NGC 3256 N and S (Lira et al. 2002; Pereira-Santaella et al. 2011; Sakamoto et al. 2014; Emonts et al. 2014; Ohyama et al. 2015); ESO 320-G030 (Pereira-Santaella et al. 2016); Arp 220 W (Barcos-Muñoz et al. 2018); and NGC 6240 (Saito et al. 2018). The crosses at the lower right corners represent the typical error bars of the points. The black lines in the left panel correspond to mass loading factors, $\eta=\dot{M} / \mathrm{SFR}$, of 1 and 10 . The dashed orange line in the middle panel marks the total momentum injected by SNe as a function of the SFR. The dashed green lines indicate the $L(\mathrm{SFR}) / c$ ratio and ten times this value. The dashed orange lines in the right panel indicate the $L_{\mathrm{out}}=a \times L_{\mathrm{SNe}}$ with $a=1,0.1$, and 0.01 as function of the SFR. The solid red lines in the middle and right panels are the best linear fits to the star-forming objects.

In this figure, we include local U/LIRGs with spatially resolved observations (filled symbols) as well as the lower luminosity starbursts compiled by Cicone et al. $(2014)^{2}$. In total, we include observations for seven ULIRG nuclei, five LIRG nuclei, and four starbursts. For the two nuclei classified as AGN, we derive the nuclear SFR from their IR luminosity after subtracting the AGN contribution (see García-Burillo et al. 2015; Ohyama et al. 2015).

The five new ULIRG nuclei (encircled stars in this figure) have mass loading factors, $\eta, \sim 0.8-2$ (see Table 9). These are similar to those observed in local starburst galaxies, which are typically lower than 2-3 (e.g., Bolatto et al. 2013; Cicone et al.

2 For NGC 3256, we use the newer observations presented by Sakamoto et al. (2014), which distinguish between the northern and southern nuclei, instead of the Sakamoto et al. (2006) data used by Cicone et al. (2014).
2014; Salak et al. 2016). This suggests that the outflows in these ULIRGs are also powered by SF.

To further investigate the energy source, in Table 9, we list the ratios between the kinetic luminosity and momentum rates of the outflows and the total energy and momentum injected by supernovae $(\mathrm{SNe})$, respectively. We assume that the $\mathrm{SNe}$ total energy and momentum are upper limits on the energy and momentum that the starburst can inject into the outflow (independent of the launching mechanism; see Sect. 4.3). For all the galaxies, both the energy and momentum in the molecular outflowing gas are lower than those produced by SNe. Although this does not imply an SF origin, we cannot rule out the SF origin based on the energy or momentum of these outflows.

Molecular outflows from AGN usually have maximum velocities up to $\sim 1000 \mathrm{~km} \mathrm{~s}^{-1}$ (e.g., Cicone et al. 2014; Veilleux et al. 2017), which are higher than those due to SF (a few hundreds of $\mathrm{km} \mathrm{s}^{-1}$ ). We found the maximum outflow velocities 
in IRAS $12112 \mathrm{NE}$ and IRAS $14348 \mathrm{SW}\left(\sim 700-800 \mathrm{~km} \mathrm{~s}^{-1}\right)$. These are not as high as those observed in other AGN, but are 1.5-2 times higher than in the rest of our sample and might indicate an AGN-powered outflow in these objects. However, there are molecular outflows detected in more nearby starbursts that also reach these high velocities (e.g., Sakamoto et al. 2014). Therefore, the velocities of the outflows in these ULIRGs are not high enough to claim an AGN origin.

Similarly, the orientation of the outflow gives information on its origin. Outflows produced by starbursts tend to be perpendicular to the disk of the galaxy where it is easier for the gas to escape. On the contrary, the angle of AGN outflows is, in principle, independent of the disk orientation (e.g., Pjanka et al. 2017). We found that the PA of these outflows are compatible with being perpendicular to the disk (i.e., possible SF origin) except for IRAS 14348 SW (i.e., possible AGN origin; see Table 5).

In summary, the mass, energy, momentum, velocity, and geometry of these outflows seem to be compatible with those expected for a SF-powered outflow. The only exception could be the outflow of IRAS 14348 SW. This outflow has a relatively high velocity compared to the others and also a different geometry, so it might be powered by an AGN. X-ray observations also suggest the presence of a Compton-thick AGN, although the bolometric luminosity of this AGN seems to be $<10 \%$ of the total IR luminosity (Iwasawa et al. 2011) and would not be able to produce the observed outflow. Therefore, since there is no clear evidence for an AGN origin, we assumed a SF origin in this case too.

\subsection{Outflow effects on the star formation}

The nuclear outflow depletion times are 15-80 Myr, which are comparable to those found in other ULIRGs (Cicone et al. 2014; García-Burillo et al. 2015; González-Alfonso et al. 2017). These times do not include the possible inflow of molecular gas into the nuclear region. However, between 70 and $90 \%$ of the molecular gas is already in these central regions (see Tables 3 and 6), so we do not expect significant molecular inflows to occur. Inflows of atomic gas might be present too, but there are no spatially resolved $\mathrm{HI}$ observations available for these objects to infer the atomic gas distribution. In addition, we have to take into account that most of the outflowing gas ( $\sim 60-80 \%$; Table 8$)$ will not escape the gravitational potential of these systems and will become available to form new stars in the future. We can estimate how long it will take for the outflowing gas to rain back into the system from the average outflow velocity, the outflow radius, and the escape velocity (Tables 7 and 8). From the escape velocity we obtain the gravitational parameter, $\mu=\mathrm{GM}$, using the relation

$\mu=\frac{1}{2} \times r_{\mathrm{esc}} \times v_{\mathrm{esc}^{2}}$,

where $v_{\text {esc }}$ and $r_{\text {esc }}$ are the escape velocity and the radius at which it is calculated, respectively. Then, assuming that the outflowing gas moves radially and that it is not affected by any dynamical friction, the equations of motion are

$$
\begin{aligned}
& \frac{\mathrm{d} r}{\mathrm{~d} t}=v, \\
& \frac{\mathrm{d} v}{\mathrm{~d} t}=-\frac{\mu}{r^{2}},
\end{aligned}
$$

with the initial conditions $t_{0}=t_{\mathrm{dyn}}, r_{0}=R_{\text {out }}$, and $v_{0}=v_{\text {out }}$. Integrating these equations numerically, we can determine when $r$

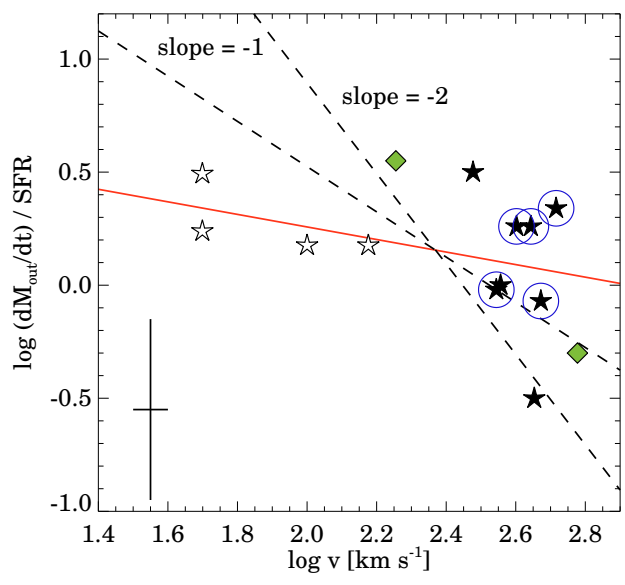

Fig. 14. Mass loading factor vs. outflow velocity. Only outflows powered by SF are plotted in this figure. Galaxy symbols are as in Fig. 13. The dashed black lines are the best fits with fixed slopes of -1 and -2 (see Sect. 4.3). The red line is the best linear fit.

becomes 0 , and obtain an estimate of the outflow cycle duration. By doing this, we find cycle durations of 5-10 Myr (these can be shorter if the dynamical friction is important). Therefore, even if the outflow depletion times are slightly shorter than the SF depletion times ( $M_{\text {tot }} / \mathrm{SFR} \sim 30-80 \mathrm{Myr}$ ), the outflowing gas will return to the starburst region after a few mega-years where it will be available again to form stars. In consequence, the main effects of these outflows are to delay the formation of stars in the nuclear starbursts and to expel a fraction of the total molecular gas $(\sim 15-30 \%)$ into the intergalactic medium. However, they will not completely quench the nuclear star formation.

Walter et al. (2017) suggested that the molecular outflow detected in the low-luminosity starburst galaxy NGC 253 is accelerating at a rate of $1 \mathrm{~km} \mathrm{~s}^{-1} \mathrm{pc}^{-1}$ when observed at $30 \mathrm{pc}$ resolution. For these ULIRGs, we find that the higher velocity outflowing molecular gas is not located farther from the nucleus than the lower velocity gas (see Fig. 5 and Appendix B). Therefore, outflow acceleration does not seem to be important for these outflows at the $\sim 500 \mathrm{pc}$ scale and will likely not affect the cycle duration and outflow effects discussed above.

\subsection{Outflow launching mechanism in starbursts}

There are two main mechanisms that can launch outflows in starbursts. Radiation pressure from young stars can deposit momentum into dust grains. Dust and gas are assumed to be dynamically coupled and, therefore, this process can increase the gas outward velocity and produce an outflow. This class of outflows is known as momentum driven (e.g., Murray et al. 2005; Thompson et al. 2015). The second mechanism is related to the energy injection into the interstellar medium (ISM) by SNe. If the gas does not cool efficiently, this energy increase translates into an adiabatic expansion of the gas, which drives the outflow. These outflows are known as energy driven (e.g., Chevalier \& Clegg 1985; Costa et al. 2014).

The scaling relation between the mass loading factor and the outflow velocity is different for energy- and momentum-driven outflows $\left(\eta \sim v^{-2}\right.$ for energy-driven and $\eta \sim v^{-1}$ for momentumdriven; e.g., Murray et al. 2005). Cicone et al. (2014) found a slope of -1 for this relation and suggested that the molecular phase of outflows are possibly momentumdriven. However, Fig. 14 shows that, after adding the new data points, the slope of 
the $\eta$ vs. $v$ relation is shallower than -1 . The best linear fit is

$\log \eta=(0.8 \pm 0.4)-(0.3 \pm 0.2) \log v_{\text {out }}\left(\mathrm{km} \mathrm{s}^{-1}\right)$.

This does not necessarily imply that these outflows are not momentumdriven. Actually, the -1 slope for momentum-driven outflows implicitly ignores the dependency of the outflow velocity on the optical depth, $\tau_{\mathrm{FIR}}$, of the launching region. When the FIR optical depth increases, the momentum transfer from the radiation to the dust and gas can be considerably more efficient (Hopkins et al. 2013; Thompson et al. 2015). For instance, if $\tau_{\text {FIR }}>1$, the momentum boost factor, $\dot{P}_{\text {out }} /(L / c)$, can significantly exceed 2 (Thompson et al. 2015).

To test this, in the middle panel of Fig. 13 we plot the outflow momentum rate as a function of the SFR. The best linear fit to the starbursts data is

$\log \dot{P}_{\text {out }}\left(\mathrm{g} \mathrm{cm} \mathrm{s}^{-2}\right)=(32.7 \pm 0.3)+(1.5 \pm 0.2) \log \operatorname{SFR}\left(M_{\odot} \mathrm{yr}^{-1}\right)$,

which has a slope $>1$. That is, for those starbursts with the lower SFR, the momentum boost factor is approximately 1 (see also Cicone et al. 2014). However, this factor increases for objects with higher SFR up to approximately 8 . For one of these starbursts, ESO 320-G030, we measured a very high optical depth in the region launching the outflow $\gtrsim 8$ at $100 \mu \mathrm{m}$ (Pereira-Santaella et al. 2017). Therefore, higher dust opacities in the more vigorous starbursts could explain these momentum boost factors $>2$.

We also explore the possible role of $\mathrm{SNe}$ in the launching of these outflows. We plot the momentum injected by $\mathrm{SNe}$ in the middle panel of Fig. 13, which is more than a factor of ten higher than the radiation pressure. For all the starbursts, the momentum rate of their outflows is lower than the momentum due to $\mathrm{SN}$ explosions. Therefore, these outflows could be launched by $\mathrm{SNe}$. If this is the case, the momentum coupling between the SNe and the ISM seems to be more efficient at higher SFR. While for the low SFR objects, the outflows carry less than $10 \%$ of the SNe momentum, the outflows in higher SFR objects carry up to $75 \%$ of the momentum injected by $\mathrm{SNe}$.

Similarly, in the right panel of Fig. 13, we compare the kinetic luminosity of the outflows with the energy produced by SNe. The outflow kinetic luminosity represents $4-20 \%$ of the energy produced by $\mathrm{SNe}$ for the U/LIRGs, whereas for the lower luminosity starbursts, this fraction is $\lesssim 1 \%$. Therefore, if these outflows are driven by $\mathrm{SNe}$, this suggests that the coupling efficiency between the SNe and the ISM increases with increasing SFR. The best linear fit is

$\log L_{\text {out }}\left(\operatorname{erg~s}^{-1}\right)=(39.0 \pm 0.3)+(2.0 \pm 0.2) \log \operatorname{SFR}\left(M_{\odot} \mathrm{yr}^{-1}\right)$

We also note that for the AGN U/LIRGs, the observed kinetic luminosities of the outflows are $1-5 \%$ of the AGN luminosity (Cicone et al. 2014; García-Burillo et al. 2015). Thus, if $\mathrm{SNe}$ are the main drivers of outflows in starbursts, the coupling between the SN explosions and the ISM must be more efficient than for AGN, at least when the SFR is sufficiently high.

\subsection{Multi-phase outflows}

\subsubsection{Outflowing molecular gas evolution}

We measure similar outflow dynamical times, around $1 \mathrm{Myr}$, in all the galaxies. These are much shorter than the age of
Table 10. Hot molecular outflow phase.

\begin{tabular}{lcccc}
\hline \hline Object & $\begin{array}{c}v_{\text {cold H}_{2}{ }^{a}} \\
\left(\mathrm{~km} \mathrm{~s}^{-1}\right)\end{array}$ & $\begin{array}{c}v_{\text {hot H}}{ }^{b} \\
\left(\mathrm{~km} \mathrm{~s}^{-1}\right)\end{array}$ & $\begin{array}{c}M_{\mathrm{hot} \mathrm{H}_{2}{ }^{c}} \\
\left(10^{3} M_{\odot}\right)\end{array}$ & $\begin{array}{c}M_{\mathrm{hot} \mathrm{H}_{2}} / M_{\text {cold H}_{2}{ }^{d}} \\
\left(10^{-5}\right)\end{array}$ \\
\hline I12112 NE & 465 & 430 & $6.8 \pm 3.7$ & $1.3 \pm 0.7$ \\
I14348 SW & 419 & 520 & $8.4 \pm 2.2$ & $1.6 \pm 0.5$ \\
I22491 E & 325 & 320 & $5.9 \pm 1.9$ & $4.9 \pm 1.6$ \\
\hline
\end{tabular}

Notes. ${ }^{(a)}$ Cold molecular outflow velocity (see Table 6). ${ }^{(b, c)}$ Velocity and mass of the hot molecular outflowing gas (Emonts et al. 2017). ${ }^{(d)}$ Hot-to-cold molecular gas ratio in the outflows.

the star-formation burst expected in ULIRGs ( 60-100 Myr; Rodríguez Zaurín et al. 2010) and also much shorter than the outflow depletion times ( 15-80 Myr; see Sect. 3.4).

This might be connected to the evolution of the gas within the outflow. For instance, if the molecular gas is swept from the nuclear ISM, it might be able to survive only $\sim 1$ Myr in the hot gas outflow environment before the molecular gas dissociates and becomes neutral atomic gas (e.g., Decataldo et al. 2017). These dynamical times are also consistent with those measured in the molecular outflow of a local starburst observed at much higher spatial resolution (Pereira-Santaella et al. 2016; Aalto et al. 2016). Alternatively, if the outflow has a bi-conical geometry, its projected area increases proportionally to $r^{2}$ as it expands. Therefore, even if the molecular gas is not dissociated, its column density rapidly decreases with increasing $r$ and, eventually, the $\mathrm{CO}$ emission will be below the detection limit of the observations. The present data do not allow us to distinguish between these possibilities because the outflow structure is just barely spatially resolved and, therefore, it is not possible to accurately measure the radial dependency of the outflow properties. It has been suggested that molecular gas forms in the outflow (e.g., Ferrara \& Scannapieco 2016; Richings \& Faucher-Giguère 2018). If so, these observations indicate that molecular gas does not efficiently form in outflows, at least beyond $1 \mathrm{kpc}$ or after $1 \mathrm{Myr}$.

\subsubsection{Hot and cold molecular phase}

There are observations of the ionized and hot molecular phases of the outflows in I12112, I14348, and I22491 that demonstrate their multi-phase structure and suggest that transitions between the different phases are possible (Arribas et al. 2014; Emonts et al. 2017). For these galaxies, a direct comparison of the $\mathrm{CO}(2-1)$ data with the observations of the ionized phase $(\mathrm{H} \alpha)$ are not possible due to the relatively low angular resolution of the $\mathrm{H} \alpha$ data $\left(\gtrsim 1^{\prime \prime}\right)$. However, the detection of a broad $\mathrm{H} \alpha$ component indicates the presence of ionized gas in the outflow. The comparison between the cold molecular and the ionized phases of the outflow in NGC 6240 is presented by Saito et al. (2018). They show that the outflow mass is dominated by the cold molecular phase in that object.

For the hot molecular phase, we have maps at higher angular resolution (Emonts et al. 2017). This hot phase is traced by the near-IR ro-vibrational $\mathrm{H}_{2}$ transitions and is detected in three cases (I12112 NE, I14348 SW, and I22491 E). The two cases where no outflow was detected in the hot phase, I12112 SW and I14348 NE, contain the least massive of the CO outflows in our ALMA sample, and may therefore have been below the detection limit of the near-IR data. In general, there is a good agreement between the outflow velocity structures (see Figs. 2-4 of Emonts et al. 2017). Also, there is a good agreement between the 
average outflow velocities (see Table 10). Interestingly, for the hot molecular $\mathrm{H}_{2}$ gas, only the blueshifted part of the outflows was unambiguously detected. The redshifted part of the outflows, as seen in CO, may have suffered from very high obscuration in the near-IR $\mathrm{H}_{2}$ lines, although the poorer spectral resolution and lower sensitivity of the near-IR data compared to the ALMA data makes this difficult to verify.

The average hot-to-cold molecular gas mass ratio is $(2.6 \pm 1.0) \times 10^{-5}$. If we only considered the blueshifted part of the outflows, this ratio would be higher by up to a factor of about 2. These estimates are slightly lower but comparable to the ratio of $6-7 \times 10^{-5}$ observed in the outflows of local LIRGs (Emonts et al. 2014; Pereira-Santaella et al. 2016), and well within the $10^{-7}-10^{-5}$ range found for molecular gas in starburst galaxies and AGN (Dale et al. 2005). This ratio provides information on the temperature distribution of molecular gas (e.g., Pereira-Santaella et al. 2014) and the excitation of the outflowing gas (e.g., Dasyra et al. 2014; Emonts et al. 2014).

The hot-to-cold molecular gas mass ratio can also be used to obtain a rough estimate of the total outflowing mass of molecular gas when only near-IR $\mathrm{H}_{2}$ data are available. This method was used in Emonts et al. (2017) to extrapolate total molecular mass outflow rates in I12112 NE, I14348 SW, and I22491 E, as based on the near-IR $\mathrm{H}_{2}$ data alone. This resulted in mass outflow estimates that were significantly lower than those found in $\mathrm{CO}$ and $\mathrm{OH}$ surveys of starburst galaxies and ULIRGs (Sturm et al. 2011; Spoon et al. 2013; Veilleux et al. 2013; Cicone et al. 2014; González-Alfonso et al. 2017). However, our new ALMA results reveal higher molecular mass outflow rates, bringing them back into agreement with these earlier surveys. This shows the importance of directly observing the cold component of molecular outflows, and it highlights the synergy between ALMA and the James Webb Space Telescope for studying the role of molecular outflows in the evolution of galaxies.

\section{Conclusions}

We analyzed new ALMA $\mathrm{CO}(2-1)$ observations of three low- $z$ ULIRG systems $(d \sim 350 \mathrm{Mpc})$. Thanks to the high $\mathrm{S} / \mathrm{N}$ and spatial resolution of these data, we were able to study the physical properties and kinematics of the molecular gas around five out of six nuclei of these three ULIRGs. Then, we used data from the literature to investigate the properties of these outflows and their impact on the evolution of the ULIRG systems. The main results of this paper are the following:

1. We have detected fast (deprojected $v_{\text {out }} \sim 350-550 \mathrm{~km} \mathrm{~s}^{-1}$; $\left.v_{\max } \sim 500-900 \mathrm{~km} \mathrm{~s}^{-1}\right)$ massive molecular outflows $\left(M_{\text {out }}\right.$ $\left.\sim(0.3-5) \times 10^{8} M_{\odot}\right)$ in the five well-detected nuclei of these three low- $z$ ULIRGs. The outflow emission is spatially resolved and we measured deprojected outflow effective radii between $250 \mathrm{pc}$ and $1 \mathrm{kpc}$. The PA of the outflow emission is compatible with an outflow perpendicular to the rotating molecular disk in three cases. Only in one case is the outflow PA clearly not along the kinematic minor axis, suggesting a different outflow orientation.

2. The outflow dynamical times are between 0.5 and $3 \mathrm{Myr}$ and the outflow rates between 12 and $400 M_{\odot} \mathrm{yr}^{-1}$. Taking into account the nuclear SFR, the mass loading factors are 0.8 to $\sim 2$. These values are similar to those found in other local ULIRGs. The total molecular gas mass in the regions where the outflows originate is $(1-7) \times 10^{9} M_{\odot}$. Therefore, the outflow depletion times are $15-80 \mathrm{Myr}$. We also estimate that only $15-30 \%$ of the outflowing gas has $v>v_{\text {esc }}$ and will escape the gravitational potential of the nucleus.
3. We use multiple indicators to determine the power source of these molecular outflows (e.g., mass loading factor, outflow energy and momentum vs. those injected by SNe, maximum outflow velocity, geometry, etc.). For all the nuclei, the observed molecular outflows are compatible with being powered by the strong nuclear starburst.

4. The outflow depletion times are slightly shorter than the SF depletion times (30-80 Myr). However, we find that most of the outflowing molecular gas does not have enough velocity to escape the gravitational potential of the nucleus. Assuming that the outflowing gas is not affected by any dynamical friction, we estimate that most of this outflowing material will return to the molecular disk after 5-10 Myr and become available to form new stars. Therefore, the main effects of these outflows are to expel part of the total molecular gas $(\sim 15-30 \%)$ into the intergalactic medium and delay the formation of stars but, possibly, they are not completely quenching the nuclear star formation.

5.Cicone et al. (2014) suggested that outflows in starbursts are driven by the radiation pressure due to young stars (i.e., momentum-driven) based on the -1 slope of the mass loading factor vs. outflow velocity relation. After adding more points to this relation, we find a shallower slope $-(0.3 \pm 0.2)$. For momentum-driven outflows, this shallower slope can be explained if the dust optical depth increases for higher luminosity starbursts enhancing the momentum boost factor. One of the nuclear starbursts in our sample has an optical depth $\gtrsim 8$ at $100 \mu \mathrm{m}$ and might support this scenario. Alternatively, these outflows might be launched by SNe. If so, the coupling efficiency between the ISM and SNe increases with increasing SFR. For the stronger starbursts, these molecular outflows carry up to 75 and $20 \%$ of the momentum and energy injected by SNe, respectively. 6 . We explore the possible evolution of the cold molecular gas in the outflow. The relatively small sizes $(<1 \mathrm{kpc})$ and short dynamical times $(<3 \mathrm{Myr})$ of the outflows suggest that molecular gas cannot survive longer in the outflow environment or that it cannot form efficiently beyond these distances or times. The detection of other outflow phases, hot molecular and ionized, for these galaxies suggests that transformation between the different outflow gas phases might exist. Alternatively, in a uniform bi-conical outflow geometry, the CO column density will eventually be below the detection limit and explain the nondetection of the outflowing molecular gas beyond $\sim 1 \mathrm{kpc}$. New high-spatial-resolution observations of similar outflows will help to distinguish between these possibilities.

Acknowledgements. We thank the anonymous referee for useful comments and suggestions. M.P.S. acknowledges support from S.T.F.C. through grant ST/N000919/1. L.C., S.G.B., and A.L. acknowledge financial support by the Spanish MEC under grants ESP2015-68964 and AYA2016-76682-C3-2-P. This paper makes use of the following ALMA data: ADS/JAO.ALMA\# 2015.1.00263.S, ADS/JAO.ALMA\#2016.1.00170.S. ALMA is a partnership of ESO (representing its member states), NSF (USA), and NINS (Japan), together with NRC (Canada), NSC and ASIAA (Taiwan), and KASI (Republic of Korea), in cooperation with the Republic of Chile. The Joint ALMA Observatory is operated by ESO, AUI/NRAO, and NAOJ. The National Radio Astronomy Observatory is a facility of the National Science Foundation operated under cooperative agreement by Associated Universities, Inc.

\section{References}

Aalto, S., Garcia-Burillo, S., Muller, S., et al. 2012, A\&A, 537, A44 Aalto, S., Garcia-Burillo, S., Muller, S., et al. 2015, A\&A, 574, A85 Aalto, S., Costagliola, F., Muller, S., et al. 2016, A\&A, 590, A73 Alonso-Herrero, A., Esquej, P., Roche, P. F., et al. 2016, MNRAS, 455, 563 Arribas, S., Colina, L., Bellocchi, E., Maiolino, R., \& Villar-Martín, M. 2014, A\&A, 568, A14

Barcos-Muñoz, L., Aalto, S., Thompson, T. A., et al. 2018, ApJ, 853, L28 
Bellocchi, E., Arribas, S., Colina, L., \& Miralles-Caballero, D. 2013, A\&A, 557, A59

Bolatto, A. D., Wolfire, M., \& Leroy, A. K. 2013, ARA\&A, 51, 207

Briggs, D. S. 1995, PhD Thesis, New Mexico Institute of Mining and Technology

Cazzoli, S., Arribas, S., Maiolino, R., \& Colina, L. 2016, A\&A, 590, A125

Chevalier, R. A., \& Clegg, A. W. 1985, Nature, 317, 44

Chu, J. K., Sanders, D. B., Larson, K. L., et al. 2017, ApJS, 229, 25

Chung, A., Yun, M. S., Naraynan, G., Heyer, M., \& Erickson, N. R. 2011, ApJ, 732, L15

Cicone, C., Maiolino, R., Sturm, E., et al. 2014, A\&A, 562, A21

Colina, L., Arribas, S., Borne, K. D., \& Monreal, A. 2000, ApJ, 533, L9

Condon, J. J., Helou, G., Sanders, D. B., \& Soifer, B. T. 1990, ApJS, 73, 359

Condon, J. J., Huang, Z.-P., Yin, Q. F., \& Thuan, T. X. 1991, ApJ, 378, 65

Costa, T., Sijacki, D., \& Haehnelt, M. G. 2014, MNRAS, 444, 2355

Courteau, S. 1997, AJ, 114, 2402

Dale, D. A., Sheth, K., Helou, G., Regan, M. W., \& Hüttemeister, S. 2005, AJ, 129,2197

Dasyra, K. M., Combes, F., Novak, G. S., et al. 2014, A\&A, 565, A46

Dasyra, K. M., Bostrom, A. C., Combes, F., \& Vlahakis, N. 2015, ApJ, 815, 34

Dasyra, K. M., Combes, F., Oosterloo, T., et al. 2016, A\&A, 595, L7

Decataldo, D., Ferrara, A., Pallottini, A., Gallerani, S., \& Vallini, L. 2017, MNRAS, 471, 4476

Emonts, B. H. C., Piqueras-López, J., Colina, L., et al. 2014, A\&A, 572, A40

Emonts, B. H. C., Colina, L., Piqueras-López, J., et al. 2017, A\&A, 607, A116

Evans, A. S., Mazzarella, J. M., Surace, J. A., \& Sanders, D. B. 2002, ApJ, 580, 749

Farrah, D., Afonso, J., Efstathiou, A., et al. 2003, MNRAS, 343, 585

Ferrara, A., \& Scannapieco, E. 2016, ApJ, 833, 46

Feruglio, C., Maiolino, R., Piconcelli, E., et al. 2010, A\&A, 518, L155

Fischer, J., Sturm, E., González-Alfonso, E., et al. 2010, A\&A, 518, L41

García-Burillo, S., Combes, F., Usero, A., et al. 2015, A\&A, 580, A35

García-Marín, M., Colina, L., Arribas, S., Alonso-Herrero, A., \& Mediavilla, E. 2006, ApJ, 650, 850

García-Marín, M., Colina, L., \& Arribas, S. 2009a, A\&A, 505, 1017

García-Marín, M., Colina, L., Arribas, S., \& Monreal-Ibero, A. 2009b, A\&A, 505,1319

González-Alfonso, E., Fischer, J., Spoon, H. W. W., et al. 2017, ApJ, 836, 11

Hill, M. J., \& Zakamska, N. L. 2014, MNRAS, 439, 2701

Hopkins, P. F., Quataert, E., \& Murray, N. 2012, MNRAS, 421, 3522

Hopkins, P. F., Kereš, D., Murray, N., et al. 2013, MNRAS, 433, 78

Imanishi, M., Nakanishi, K., \& Izumi, T. 2016, AJ, 152, 218

Imanishi, M., Nakanishi, K., \& Izumi, T. 2018, ApJ, 856, 143

Iwasawa, K., Sanders, D. B., Teng, S. H., et al. 2011, A\&A, 529, A106

Kennicutt, R. C., \& Evans, N. J. 2012, ARA\&A, 50, 531

Kim, C.-G., \& Ostriker, E. C. 2015, ApJ, 802, 99

Kroupa, P. 2001, MNRAS, 322, 231

Law, D. R., Steidel, C. C., Erb, D. K., et al. 2009, ApJ, 697, 2057

Leitherer, C., Schaerer, D., Goldader, J. D., et al. 1999, ApJS, 123, 3

Lira, P., Ward, M., Zezas, A., Alonso-Herrero, A., \& Ueno, S. 2002, MNRAS, 330, 259

Martí-Vidal, I., Vlemmings, W. H. T., Muller, S., \& Casey, S. 2014, A\&A, 563, A136

McMullin, J. P., Waters, B., Schiebel, D., Young, W., \& Golap, K. 2007, in Astronomical Data Analysis Software and Systems XVI, eds. R. A. Shaw, F. Hill, \& D. J. Bell, ASP Conf. Ser., 376, 127
Murphy, E. J., Condon, J. J., Schinnerer, E., et al. 2011, ApJ, 737, 67

Murray, N., Quataert, E., \& Thompson, T. A. 2005, ApJ, 618, 569

Narayanan, D., Krumholz, M., Ostriker, E. C., \& Hernquist, L. 2011, MNRAS, 418,664

Nardini, E., Risaliti, G., Watabe, Y., Salvati, M., \& Sani, E. 2010, MNRAS, 405, 2505

Nelson, D., Genel, S., Vogelsberger, M., et al. 2015, MNRAS, 448, 59

Ohyama, Y., Terashima, Y., \& Sakamoto, K. 2015, ApJ, 805, 162

Papadopoulos, P. P., van der Werf, P., Xilouris, E., Isaak, K. G., \& Gao, Y. 2012, ApJ, 751, 10

Pereira-Santaella, M., Alonso-Herrero, A., Santos-Lleo, M., et al. 2011, A\&A, 535, A93

Pereira-Santaella, M., Spinoglio, L., van der Werf, P. P., \& Piqueras López J. 2014, A\&A, 566, A49

Pereira-Santaella, M., Colina, L., Alonso-Herrero, A., et al. 2015, MNRAS, 454, 3679

Pereira-Santaella, M., Colina, L., García-Burillo, S., et al. 2016, A\&A, 594, A81

Pereira-Santaella, M., González-Alfonso, E., Usero, A., et al. 2017, A\&A, 601, L3

Piqueras López, J., Colina, L., Arribas, S., Alonso-Herrero, A., \& Bedregal, A. G. 2012, A\&A, 546, A64

Piqueras López, J., Colina, L., Arribas, S., \& Alonso-Herrero, A. 2013, A\&A, 553, A85

Piqueras López, J., Colina, L., Arribas, S., Pereira-Santaella, M., \& AlonsoHerrero, A. 2016, A\&A, 590, A67

Pjanka, P., Greene, J. E., Seth, A. C., et al. 2017, ApJ, 844, 165

Richings, A. J., \& Faucher-Giguère, C.-A. 2018, MNRAS, 474, 3673

Rodríguez Zaurín, J., Tadhunter, C. N., \& González Delgado R. M. 2010, MNRAS, 403, 1317

Rupke, D. S., Veilleux, S., \& Sanders, D. B. 2005, ApJ, 632, 751

Saito, T., Iono, D., Xu, C. K., et al. 2017, ApJ, 835, 174

Saito, T., Iono, D., Ueda, J., et al. 2018, MNRAS, 475, L52

Sakamoto, K., Ho, P. T. P., \& Peck, A. B. 2006, ApJ, 644, 862

Sakamoto, K., Aalto, S., Combes, F., Evans, A., \& Peck, A. 2014, ApJ, 797, 90

Salak, D., Nakai, N., Hatakeyama, T., \& Miyamoto, Y. 2016, ApJ, 823, 68

Scannapieco, C., Wadepuhl, M., Parry, O. H., et al. 2012, MNRAS, 423, 1726

Schaye, J., Crain, R. A., Bower, R. G., et al. 2015, MNRAS, 446, 521

Spoon, H. W. W., Farrah, D., Lebouteiller, V., et al. 2013, ApJ, 775, 127

Sturm, E., González-Alfonso, E., Veilleux, S., et al. 2011, ApJ, 733, L16

Thompson, T. A., Fabian, A. C., Quataert, E., \& Murray, N. 2015, MNRAS, 449,147

Veilleux, S., Kim, D.-C., \& Sanders, D. B. 1999, ApJ, 522, 113

Veilleux, S., Kim, D., \& Sanders, D. B. 2002, ApJS, 143, 315

Veilleux, S., Rupke, D. S. N., Kim, D.-C., et al. 2009, ApJS, 182, 628

Veilleux, S., Meléndez, M., Sturm, E., et al. 2013, ApJ, 776, 27

Veilleux, S., Bolatto, A., Tombesi, F., et al. 2017, ApJ, 843, 18

Walter, F., Bolatto, A. D., Leroy, A. K., et al. 2017, ApJ, 835, 265

Westmoquette, M. S., Clements, D. L., Bendo, G. J., \& Khan, S. A. 2012, MNRAS, 424, 416

Wilson, C. D., Petitpas, G. R., Iono, D., et al. 2008, ApJS, 178, 189 


\section{Appendix A: Continuum visibility fits}

Figure A.1 compares the real part of the continuum visibilities for each source with the best-fit model discussed in Sect. 3.1.2. To obtain these visibilities, we shifted the phase center to the coordinates obtained by UVMULTIFIT. For the objects with two continuum sources in the field of view, we subtracted the model of the source that is not plotted in that panel.
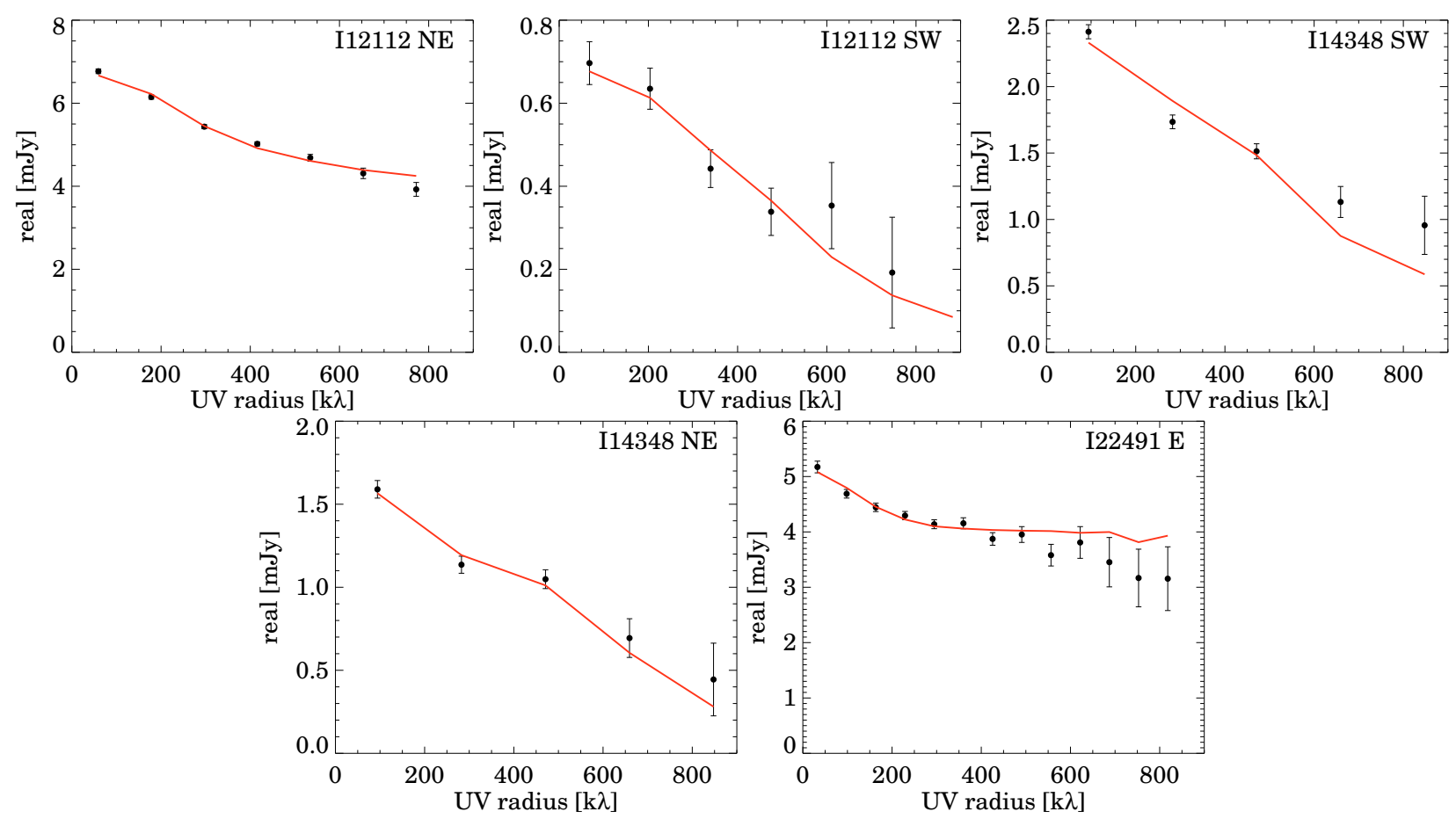

Fig. A.1. Real part of the $248 \mathrm{GHz}$ continuum visibilities as function of the $u v$ radius. The red line is the best-fit model discussed in Sect. 3.1 .2 (see also Table 4). 


\section{Appendix B: $C O(2-1)$ channel maps}

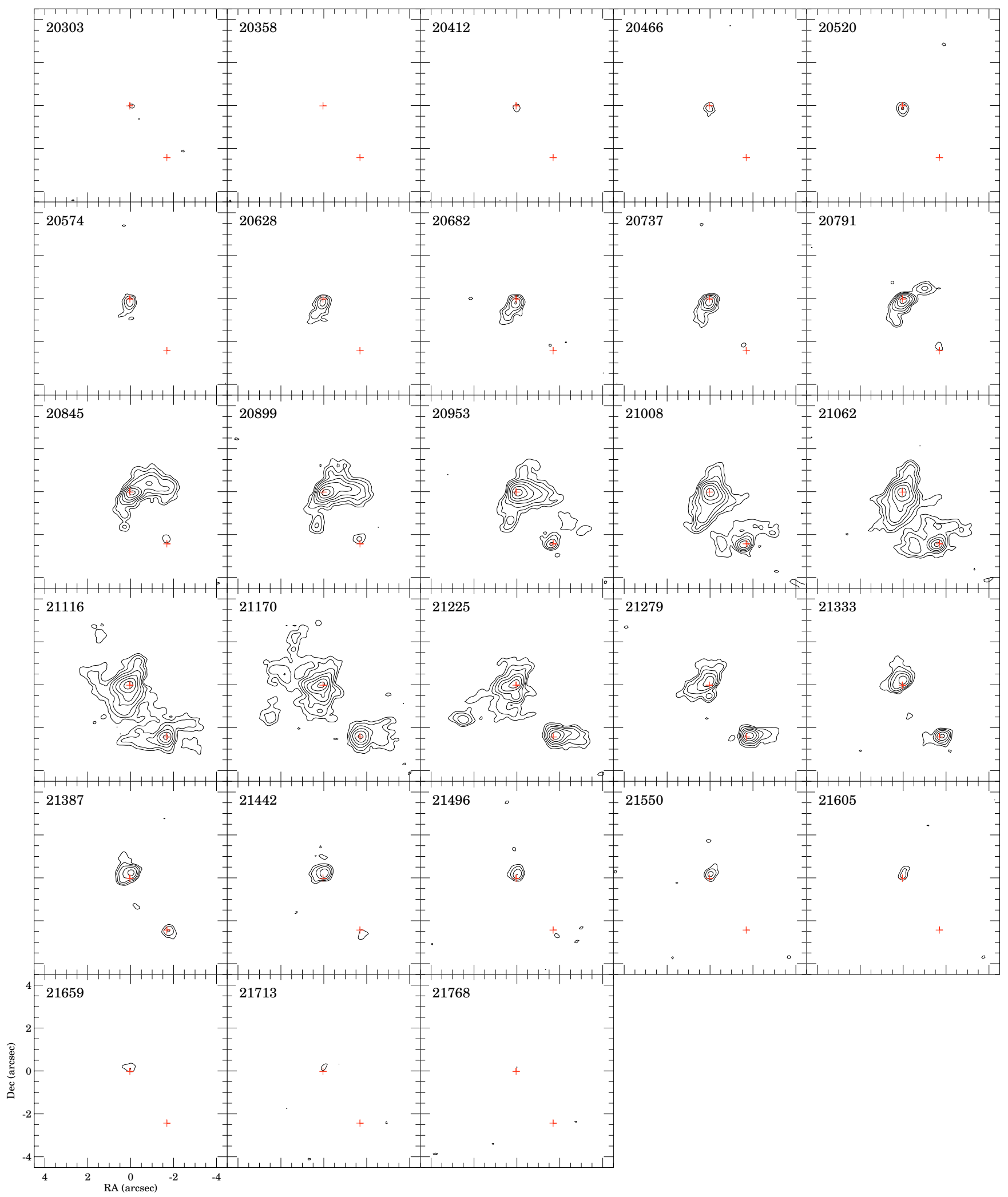

Fig. B.1. Channel maps showing the $\mathrm{CO}(2-1) 230.5 \mathrm{GHz}$ emission in IRAS $12112+0305$. Each panel shows the emission averaged over $39 \mathrm{MHz}$ $\left(\sim 50 \mathrm{~km} \mathrm{~s}^{-1}\right)$ channels. The contours correspond to $(3,6,12,24,48,96,192) \times \sigma$ and $\sigma$ is the rms measured in each channel $\left(180-260 \mu \mathrm{Jy}\right.$ beam $\left.{ }^{-1}\right)$ for this system. The relativistic LSRK velocity is indicated in each panel. The red crosses mark the location of the nuclei listed in Table 1. 
M. Pereira-Santaella et al.: Molecular outflows in ULIRGs

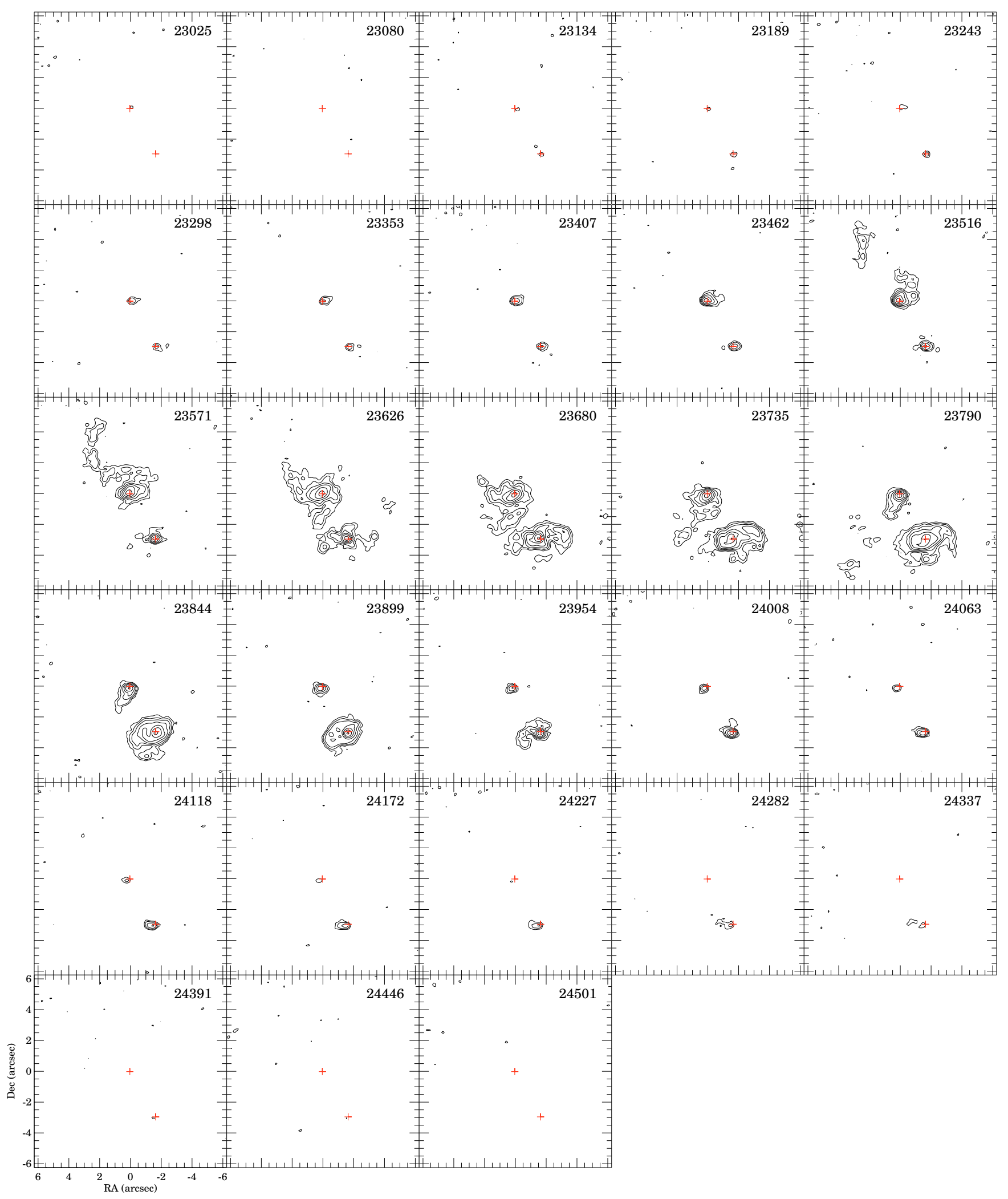

Fig. B.2. Same as Fig. B.1 but for IRAS $14348-1447$. For this system $\sigma=200-300 \mu \mathrm{Jy}_{\text {beam }}{ }^{-1}$ depending on the channel. 
A\&A 616, A171 (2018)

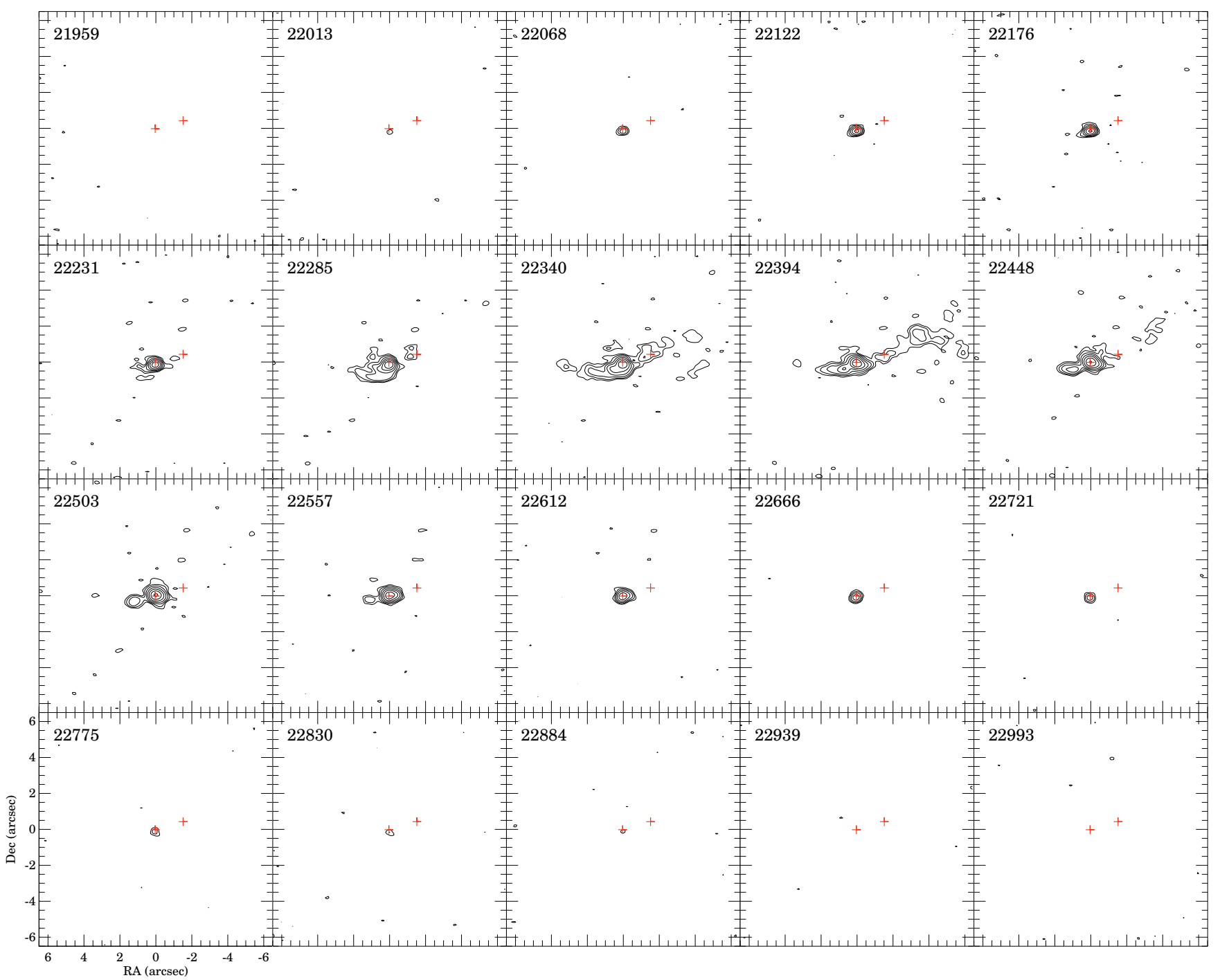

Fig. B.3. Same as Fig. B.1 but for IRAS 22491-1808. For this system $\sigma=250-400 \mu \mathrm{Jy}_{\text {beam }}{ }^{-1}$ depending on the channel. 